\title{
Gelfand-Graev characters of the finite unitary groups
}

\author{
Nathaniel Thiem \\ University of Colorado at Boulder \\ thiemn@colorado.edu
}

\author{
C. Ryan Vinroot \\ College of William and Mary \\ vinroot@math.wm.edu
}

Submitted: Aug 12, 2009; Accepted: Nov 25, 2009; Published: Nov 30, 2009
Mathematics Subject Classification: 20C33, 05E05

\begin{abstract}
Gelfand-Graev characters and their degenerate counterparts have an important role in the representation theory of finite groups of Lie type. Using a characteristic map to translate the character theory of the finite unitary groups into the language of symmetric functions, we study degenerate Gelfand-Graev characters of the finite unitary group from a combinatorial point of view. In particular, we give the values of Gelfand-Graev characters at arbitrary elements, recover the decomposition multiplicities of degenerate Gelfand-Graev characters in terms of tableau combinatorics, and conclude with some multiplicity consequences.
\end{abstract}

\section{Introduction}

Gelfand-Graev modules have played an important role in the representation theory of finite groups of Lie type $[4,7,22]$. In particular, if $G$ is a finite group of Lie type, then Gelfand-Graev modules of $G$ both contain cuspidal representations of $G$ as submodules, and have a multiplicity free decomposition into irreducible $G$-modules. Thus, GelfandGraev modules can give constructions for some cuspidal $G$-modules. This paper uses a combinatorial correspondence between characters and symmetric functions (as described in [23]) to examine the Gelfand-Graev character and its degenerate relatives for the finite unitary group.

Let $B^{<}$be a maximal unipotent subgroup of a finite group of Lie type $G$. Then the Gelfand-Graev character $\Gamma$ of $G$ is the character obtained by inducing a generic linear character from $B^{<}$to $G$. The degenerate Gelfand-Graev characters of $G$ are obtained by inducing arbitrary linear characters. In the case $\operatorname{GL}\left(n, \mathbb{F}_{q}\right)$, Zelevinsky [27] described the multiplicities of irreducible characters in degenerate Gelfand-Graev characters by counting multi-tableaux of specified shape and weight. It is the goal of this paper to describe the degenerate Gelfand-Graev characters of the finite unitary groups in a similar manner using tableau combinatorics. In [27], Zelevinsky obtained the result that every irreducible 
character of $\operatorname{GL}\left(n, \mathbb{F}_{q}\right)$ appears with multiplicity one in some degenerate Gelfand-Graev character. It is known that this multiplicity one result is not true in a general finite group of Lie type, and in fact there are characters which do not appear in any degenerate Gelfand-Graev character in the general case. This result was illustrated by Srinivasan [20] in the case of the symplectic group $\operatorname{Sp}\left(4, \mathbb{F}_{q}\right)$, and the work of Kotlar [11] gives a geometric description of the irreducible characters which appear in some degenerate Gelfand-Graev character in general type. In the finite unitary case, we give a combinatorial description of which irreducible characters appear in some degenerate Gelfand-Graev character, as well as a combinatorial description of a large family of characters which appear with multiplicity one.

In Section 2, we describe the main combinatorial tool which we use for calculations, which is the characteristic map of the finite unitary group, and we follow the development given in [23]. This map translates the Deligne-Lusztig theory of the finite unitary group into symmetric functions, which thus translates calculations in representation theory into algebraic combinatorics. Some of the results in this paper could be obtained, albeit in a different formulation, by applying Harish-Chandra induction and the representation theory of Weyl groups. However, this approach would not lead us to some of the combinatorics which we study here. For example, we naturally arrive at battery tableaux, which are interesting combinatorial objects in their own right. Also, our more classical approach gives rise to useful identities in symmetric function theory, such as our Lemma 4.2 .

Section 3 examines the (non-degenerate) Gelfand-Graev character. We use a remarkable formula for the character values of the Gelfand-Graev character of $\operatorname{GL}\left(n, \mathbb{F}_{q}\right)$, given in Theorem 3.2 (for an elementary proof see [9]), to obtain the corresponding formula for $\mathrm{U}\left(n, \mathbb{F}_{q^{2}}\right)$ in Corollary 3.1, which states that if $\Gamma_{(n)}$ is the Gelfand-Graev character of $\mathrm{U}\left(n, \mathbb{F}_{q^{2}}\right)$, and $g \in \mathrm{U}\left(n, \mathbb{F}_{q^{2}}\right)$, then

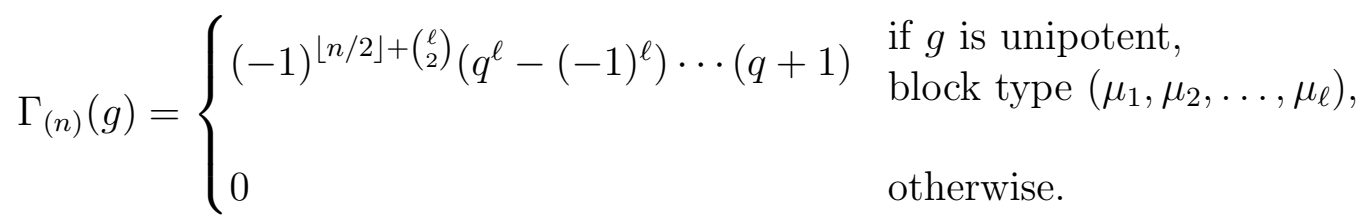

When compared to the original $\mathrm{GL}\left(n, \mathbb{F}_{q}\right)$ version of this formula given in Theorem 3.2, Corollary 3.1 could be seen as another occurrence of "Ennola duality." Although the proof of Corollary 3.1 is a fairly straightforward application of the characteristic map, we have not found it stated in any of the literature. We also note that we have applied Corollary 3.1 in another paper, to obtain [24, Theorem 4.4].

Section 4 computes the decomposition of degenerate Gelfand-Graev characters in a fashion analogous to [27], using tableau combinatorics. The main result is Theorem 4.4, which may be summarized as saying that the degenerate Gelfand-Graev character $\Gamma_{(k, \nu)}$ of $\mathrm{U}\left(n, \mathbb{F}_{q^{2}}\right)$ decomposes as

$$
\Gamma_{(k, \nu)}=\sum_{\boldsymbol{\lambda}} m_{\boldsymbol{\lambda}} \chi^{\boldsymbol{\lambda}}
$$

where $\boldsymbol{\lambda}$ is a multipartition and $m_{\boldsymbol{\lambda}}$ is a nonnegative integer obtained by counting 'battery 
tableaux' of a given weight and shape. In the process of proving Theorem 4.4, we obtain some combinatorial Pieri-type formulas (Lemma 4.2), decompositions of induced characters from $\operatorname{GL}\left(n, \mathbb{F}_{q^{2}}\right)$ to $\mathrm{U}\left(2 n, \mathbb{F}_{q^{2}}\right)$ (Theorem 4.1 and Theorem 4.2), and a description of all of the cuspidal characters of the finite unitary groups (Theorem 4.3).

Section 5 concludes with a discussion of the multiplicity implications of Section 4 . In particular, in Theorem 5.2 we give combinatorial conditions on multipartitions $\boldsymbol{\lambda}$ which guarantee that the irreducible character $\chi^{\boldsymbol{\lambda}}$ appears with multiplicity one in some degenerate Gelfand-Graev character. Our Theorem 5.2 improves a multiplicity one result of Ohmori [18].

Another question one might ask is how the generalized Gelfand-Graev representations of the finite unitary group decompose. Generalized Gelfand-Graev representations, which were defined by Kawanaka in [10], are obtained by inducing certain irreducible representations (not necessarily one dimensional) from a unipotent subgroup. Rainbolt studies the generalized Gelfand-Graev representations of $\mathrm{U}\left(3, \mathbb{F}_{q^{2}}\right)$ in [19], but in the general case they seem to be significantly more complicated than the degenerate Gelfand-Graev representations.

Acknowledgements. We would like to thank G. Malle for suggesting the questions that led to the results in Section 5, S. Assaf for a helpful discussion regarding Section 5.1, T. Lam for helping us connect Lemma 4.2 to the literature, and anonymous referees for helpful comments.

\section{Preliminaries}

\subsection{Partitions}

Let

$$
\mathcal{P}=\bigcup_{n \geqslant 0} \mathcal{P}_{n}, \quad \text { where } \quad \mathcal{P}_{n}=\{\text { partitions of } n\}
$$

For $\nu=\left(\nu_{1}, \nu_{2}, \ldots, \nu_{l}\right) \in \mathcal{P}_{n}$, where $\nu_{1} \geqslant \nu_{2} \geqslant \cdots \geqslant \nu_{\ell}>0$, the length $\ell(\nu)$ of $\nu$ is the number of parts $l$, and the size $|\nu|$ of $\nu$ is the sum of the parts $n$. Let $\nu^{\prime}$ denote the conjugate of the partition $\nu$. We also write

$$
\nu=\left(1^{m_{1}(\nu)} 2^{m_{2}(\nu)} \cdots\right), \quad \text { where } \quad m_{i}(\nu)=\left|\left\{j \in \mathbb{Z}_{\geqslant 1} \mid \nu_{j}=i\right\}\right| .
$$

We will denote the unique element of $\mathcal{P}_{0}$ by $\emptyset$ or $(0)$, which is the empty partition, or the unique partition of 0 . For any $\nu \in \mathcal{P}$, define $n(\nu)$ to be

$$
n(\nu)=\sum_{i}(i-1) \nu_{i}
$$

If $\mu, \nu \in \mathcal{P}$, we define $\mu \cup \nu \in \mathcal{P}$ to be the partition of size $|\mu|+|\nu|$ whose set of parts is the union of the parts of $\mu$ and $\nu$. For $k \in \mathbb{Z}_{\geqslant 1}$, let $k \nu=\left(k \nu_{1}, k \nu_{2}, \ldots\right)$, and if every part of $\nu$ is divisible by $k$, then we let $\nu / k=\left(\nu_{1} / k, \nu_{2} / k, \ldots\right)$. A partition $\nu$ is even if $\nu_{i}$ is even for $1 \leqslant i \leqslant \ell(\nu)$. 


\subsection{The ring of symmetric functions}

Let $X=\left\{X_{1}, X_{2}, \ldots\right\}$ be an infinite set of variables and let

$$
\Lambda(X)=\mathbb{C}\left[p_{1}(X), p_{2}(X), \ldots\right], \quad \text { where } \quad p_{k}(X)=X_{1}^{k}+X_{2}^{k}+\cdots,
$$

be the graded $\mathbb{C}$-algebra of symmetric functions in the variables $\left\{X_{1}, X_{2}, \ldots\right\}$. For a partition $\nu=\left(\nu_{1}, \nu_{2}, \ldots, \nu_{\ell}\right) \in \mathcal{P}$, the power-sum symmetric function $p_{\nu}(X)$ is

$$
p_{\nu}(X)=p_{\nu_{1}}(X) p_{\nu_{2}}(X) \cdots p_{\nu_{\ell}}(X) .
$$

The irreducible characters $\omega^{\lambda}$ of $S_{n}$ are indexed by $\lambda \in \mathcal{P}_{n}$. Let $\omega^{\lambda}(\nu)$ be the value of $\omega^{\lambda}$ on a permutation with cycle type $\nu$.

The Schur function $s_{\lambda}(X)$ is given by

$$
s_{\lambda}(X)=\sum_{\nu \in \mathcal{P}_{|\lambda|}} \omega^{\lambda}(\nu) z_{\nu}^{-1} p_{\nu}(X), \quad \text { where } \quad z_{\nu}=\prod_{i \geqslant 1} i^{m_{i}} m_{i} !
$$

is the order of the centralizer in $S_{n}$ of the conjugacy class corresponding to the partition $\nu=\left(1^{m_{1}} 2^{m_{2}} \ldots\right) \in \mathcal{P}_{n}$.

Fix $t \in \mathbb{C}^{\times}$. For $\mu \in \mathcal{P}$, the Hall-Littlewood symmetric function $P_{\mu}(X ; t)$ is given by

$$
s_{\lambda}(X)=\sum_{\mu \in \mathcal{P}_{|\lambda|}} K_{\lambda \mu}(t) P_{\mu}(X ; t),
$$

where $K_{\lambda \mu}(t)$ is the Kostka-Foulkes polynomial (as in [17, III.6]). For $\nu, \mu \in \mathcal{P}_{n}$, the classical Green function $Q_{\nu}^{\mu}(t)$ is given by

$$
p_{\nu}(X)=\sum_{\mu \in \mathcal{P}_{|\nu|}} Q_{\nu}^{\mu}\left(t^{-1}\right) t^{n(\mu)} P_{\mu}(X ; t) .
$$

As a graded ring,

$$
\begin{aligned}
\Lambda(X) & =\mathbb{C}-\operatorname{span}\left\{p_{\nu}(X) \mid \nu \in \mathcal{P}\right\} \\
& =\mathbb{C}-\operatorname{span}\left\{s_{\lambda}(X) \mid \lambda \in \mathcal{P}\right\} \\
& =\mathbb{C}-\operatorname{span}\left\{P_{\mu}(X ; t) \mid \mu \in \mathcal{P}\right\},
\end{aligned}
$$

with change of bases given in (2.1), (2.2), and (2.3).

We will also use several product formulas in the ring of symmetric functions. The usual product on Schur functions

$$
s_{\nu} s_{\mu}=\sum_{\lambda \in \mathcal{P}} c_{\nu \mu}^{\lambda} s_{\lambda}
$$

gives us the Littlewood-Richardson coefficients $c_{\nu \mu}^{\lambda}$. The plethysm of $p_{\nu}$ with $p_{k}$ is

$$
p_{\nu} \circ p_{k}=p_{k \nu} .
$$


Thus, we can consider the nonnegative integers $c_{\lambda}^{\gamma}$ given by

$$
s_{\lambda} \circ p_{k}=\sum_{\nu \in \mathcal{P}_{|\lambda|}} \frac{\omega^{\lambda}(\nu)}{z_{\nu}} p_{k \nu}=\sum_{\gamma \in \mathcal{P}_{k|\lambda|}} c_{\lambda}^{\gamma} s_{\gamma} .
$$

Chen, Garsia, and Remmel [2] give a combinatorial algorithm for computing the coefficients $c_{\lambda}^{\gamma}$. We will use the case $k=2$ in Section 4.4 .

Remark. The unipotent characters $\chi^{\tilde{\lambda}}$ of $\operatorname{GL}\left(n, \mathbb{F}_{q^{2}}\right)$ are indexed by partitions $\tilde{\lambda}$ of $n$ and the unipotent characters $\chi^{\gamma}$ of $\mathrm{U}\left(2 n, \mathbb{F}_{q^{2}}\right)$ are indexed by partitions $\gamma$ of $2 n$. It will follow from Theorem 4.2 that

$$
R_{\mathrm{GL}\left(n, \mathbb{F}_{q^{2}}\right)}^{\mathrm{U}\left(2 n, \mathbb{F}_{q^{2}}\right)}\left(\chi^{\tilde{\lambda}}\right)=\sum_{|\gamma|=2|\tilde{\lambda}|} c_{\tilde{\lambda}}^{\gamma} \chi^{\gamma}
$$

where $R_{H}^{G}$ is Harish-Chandra induction.

\subsection{The finite unitary groups}

Let $\bar{G}_{n}=\operatorname{GL}\left(n, \overline{\mathbb{F}}_{q}\right)$ be the general linear group with entries in the algebraic closure of the finite field $\mathbb{F}_{q}$ with $q$ elements.

For the Frobenius automorphisms $\tilde{F}, F, F^{\prime}: \bar{G}_{n} \rightarrow \bar{G}_{n}$ given by

$$
\begin{array}{rlr}
\tilde{F}\left(\left(a_{i j}\right)\right) & =\left(a_{i j}^{q}\right), \\
F\left(\left(a_{i j}\right)\right) & =\left(a_{j i}^{q}\right)^{-1}, & \\
F^{\prime}\left(\left(a_{i j}\right)\right) & =\left(a_{n-j, n-i}^{q}\right)^{-1}, & \text { where }\left(a_{i j}\right) \in \bar{G}_{n},
\end{array}
$$

let

$$
\begin{aligned}
& G_{n}=\bar{G}_{n}^{\tilde{F}}=\left\{a \in \bar{G}_{n} \mid \tilde{F}(a)=a\right\}, \\
& U_{n}=\bar{G}_{n}^{F}=\left\{a \in \bar{G}_{n} \mid F(a)=a\right\}, \\
& U_{n}^{\prime}=\bar{G}_{n}^{F^{\prime}}=\left\{a \in \bar{G}_{n} \mid F^{\prime}(a)=a\right\} .
\end{aligned}
$$

Then $G_{n}=\operatorname{GL}\left(n, \mathbb{F}_{q}\right)$ and $U_{n}^{\prime} \cong U_{n}$ are isomorphic to the finite unitary group $\mathrm{U}\left(n, \mathbb{F}_{q^{2}}\right)$. In fact, it follows from the Lang-Steinberg theorem that $U_{n}^{\prime}$ and $U_{n}$ are conjugate subgroups of $\bar{G}_{n}$.

For $k \in \mathbb{Z}_{\geqslant 0}$, let

$$
\tilde{T}_{(k)}=\bar{G}_{1}^{\tilde{F}^{k}} \cong \mathbb{F}_{q^{k}}^{\times} \quad \text { and } \quad T_{(k)}=\bar{G}_{1}^{F^{k}} \cong \begin{cases}\mathbb{F}_{q^{k}}^{\times} & \text {if } k \text { is even, } \\ \left\{t \in \overline{\mathbb{F}}_{q} \mid t^{q^{k}+1}=1\right\} & \text { if } k \text { is odd } .\end{cases}
$$

For every partition $\eta=\left(\eta_{1}, \eta_{2}, \ldots, \eta_{\ell}\right) \in \mathcal{P}_{n}$ let

$$
\begin{aligned}
& T_{\eta}=T_{\left(\eta_{1}\right)} \times T_{\left(\eta_{2}\right)} \times \cdots \times T_{\left(\eta_{\ell}\right)} \\
& \tilde{T}_{\eta}=\tilde{T}_{\left(\eta_{1}\right)} \times \tilde{T}_{\left(\eta_{2}\right)} \times \cdots \times \tilde{T}_{\left(\eta_{\ell}\right)} .
\end{aligned}
$$

Every maximal torus of $G_{n}$ is isomorphic to $\tilde{T}_{\eta}$ for some $\eta \in \mathcal{P}_{n}$, and every maximal torus of $U_{n}$ is isomorphic to $T_{\eta}$ for some $\eta \in \mathcal{P}_{n}$. 


\subsection{Multipartitions}

Let $F: \bar{G}_{n} \rightarrow \bar{G}_{n}$ be as in (2.6), and let $T_{(k)}^{*}=\left\{\xi: T_{(k)} \rightarrow \mathbb{C}^{\times}\right\}$be the group of multiplicative complex-valued characters of $T_{(k)}=\bar{G}_{1}^{F^{k}}$. We identify $\overline{\mathbb{F}}_{q}^{\times}$with $\bar{G}_{1}=$ $\mathrm{GL}\left(1, \overline{\mathbb{F}}_{q}\right)$. Consider

$$
\Phi=\left\{F \text {-orbits of } \overline{\mathbb{F}}_{q}^{\times}\right\},
$$

and note that $\bar{G}_{1}=\bigcup_{f \in \Phi} f=\bigcup_{k} T_{(k)}$. In particular, we may view $\bar{G}_{1}$ as a direct limit of the $T_{(k)}$ with respect to inclusion. We also have norm maps, $N_{m, k}$, whenever $k \mid m$,

$$
\begin{aligned}
N_{m, k}: T_{(m)} & \longrightarrow T_{(k)} \\
\alpha & \mapsto \prod_{i=0}^{(m / k)-1} \alpha^{(-q)^{k i}}, \quad \text { where } m, k \in \mathbb{Z}_{\geqslant 1}, k \mid m .
\end{aligned}
$$

When $k \mid m$, denote by $N_{m, k}^{*}$ the transpose of the map $N_{m, k}$, which embeds $T_{(k)}^{*}$ into $T_{(m)}^{*}$ as follows:

$$
\begin{aligned}
N_{m, k}^{*}: T_{(k)}^{*} & \longrightarrow T_{(m)}^{*} \\
\xi & \mapsto \xi \circ N_{m, k}
\end{aligned}
$$

Now, define $L$ to be the direct limit of the groups $T_{(k)}^{*}$ with respect to the maps $N_{m, k}^{*}$ :

$$
L=\underset{\lim }{\longrightarrow} T_{(m)}^{*}
$$

Since the map $F$ acts naturally on each $T_{(m)}^{*}$, it acts on their direct limit $L$. Note that we may identify the fixed points $L^{F^{m}}$ with the character group $T_{(m)}^{*}$. Let $\Theta$ be the collection of $F$-orbits on $L$ :

$$
\Theta=\{F \text {-orbits of } L\}
$$

For $\mathcal{X} \in\{\Phi, \Theta\}$, an $\mathcal{X}$-partition $\boldsymbol{\lambda}=\left(\boldsymbol{\lambda}^{\left(x_{1}\right)}, \boldsymbol{\lambda}^{\left(x_{2}\right)}, \ldots\right)$ is a sequence of partitions indexed by $\mathcal{X}$. The size of $\boldsymbol{\lambda}$ is

$$
|\boldsymbol{\lambda}|=\sum_{x \in \mathcal{X}}|x|\left|\boldsymbol{\lambda}^{(x)}\right|
$$

where $|x|$ is the size of the orbit $x$. Note that in order for $|\boldsymbol{\lambda}|$ to be finite, we need to assume that $\boldsymbol{\lambda}^{(x)}=\emptyset$ for all but finitely many $x \in \mathcal{X}$.

Let

$$
\mathcal{P}^{\mathcal{X}}=\bigcup_{n \geqslant 0} \mathcal{P}_{n}^{\mathcal{X}}, \quad \text { where } \quad \mathcal{P}_{n}^{\mathcal{X}}=\{\mathcal{X} \text {-partitions of size } n\} .
$$

For $\boldsymbol{\lambda} \in \mathcal{P}^{\mathcal{X}}$, let

$$
\ell(\boldsymbol{\lambda})=\sum_{x \in \mathcal{X}} \ell\left(\boldsymbol{\lambda}^{(x)}\right) \quad \text { and } \quad n(\boldsymbol{\lambda})=\sum_{x \in \mathcal{X}}|x| n\left(\boldsymbol{\lambda}^{(x)}\right) .
$$

The conjugate of $\boldsymbol{\lambda} \in \mathcal{P}^{\mathcal{X}}$ is the $\mathcal{X}$-partition $\boldsymbol{\lambda}^{\prime}$ defined by $\boldsymbol{\lambda}^{\prime(x)}=\left(\boldsymbol{\lambda}^{(x)}\right)^{\prime}$, and if $\boldsymbol{\mu}, \boldsymbol{\lambda} \in \mathcal{P}^{\mathcal{X}}$, then $\boldsymbol{\mu} \cup \boldsymbol{\lambda} \in \mathcal{P}^{\mathcal{X}}$ is defined by $(\boldsymbol{\mu} \cup \boldsymbol{\lambda})^{(x)}=\boldsymbol{\mu}^{(x)} \cup \boldsymbol{\lambda}^{(x)}$. 
The semisimple part $\boldsymbol{\lambda}_{s}$ of an $\mathcal{X}$-partition $\boldsymbol{\lambda}$ is the $\mathcal{X}$-partition given by

$$
\boldsymbol{\lambda}_{s}^{(x)}=\left(1^{|\boldsymbol{\lambda}(x)|}\right), \quad \text { for } x \in \mathcal{X} .
$$

For $\boldsymbol{\lambda} \in \mathcal{P}^{\mathcal{X}}$, define the set $\mathcal{P}_{s}^{\boldsymbol{\lambda}}$ by

$$
\mathcal{P}_{s}^{\lambda}=\left\{\boldsymbol{\mu} \in \mathcal{P}^{\mathcal{X}} \mid \boldsymbol{\mu}_{s}=\boldsymbol{\lambda}_{s}\right\} .
$$

The unipotent part $\boldsymbol{\lambda}_{u}$ of $\boldsymbol{\lambda}$ is the $\mathcal{X}$-partition given by

$$
\boldsymbol{\lambda}_{u}^{(\{1\})} \text { has parts }\left\{|x| \boldsymbol{\lambda}_{i}^{(x)} \mid x \in \mathcal{X}, i=1, \ldots, \ell(\boldsymbol{\lambda}(x))\right\},
$$

where $\{1\}$ is the orbit containing 1 in $\Phi$ or the trivial character in $\Theta$, and $\boldsymbol{\lambda}_{u}^{(x)}=\emptyset$ when $x \neq\{1\}$.

Note that we can think of "normal" partitions as $\mathcal{X}$-partitions $\boldsymbol{\lambda}$ that satisfy $\boldsymbol{\lambda}_{u}=\boldsymbol{\lambda}$. By a slight abuse of notation, we will sometimes interchange the multipartition $\boldsymbol{\lambda}_{u}$ and the partition $\boldsymbol{\lambda}_{u}^{(\{1\})}$. For example, $T_{\boldsymbol{\lambda}_{u}}$ will denote the torus corresponding to the partition $\lambda_{u}^{(\{1\})}$.

Given the torus $T_{\eta}, \eta=\left(\eta_{1}, \eta_{2}, \ldots, \eta_{\ell}\right) \in \mathcal{P}_{n}$, there is a natural surjection

$$
\begin{array}{ccc}
\boldsymbol{\tau}_{\Theta}:\left\{\theta=\theta_{1} \otimes \theta_{2} \otimes \cdots \otimes \theta_{\ell} \in \operatorname{Hom}\left(T_{\eta}, \mathbb{C}^{\times}\right)\right\} & \longrightarrow & \left\{\boldsymbol{\nu} \in \mathcal{P}^{\Theta} \mid \boldsymbol{\nu}_{u}^{(\{1\})}=\eta\right\} \\
\theta=\theta_{1} \otimes \theta_{2} \otimes \cdots \otimes \theta_{\ell} & \mapsto & \boldsymbol{\tau}_{\Theta}(\theta),
\end{array}
$$

where

$$
\boldsymbol{\tau}_{\Theta}(\theta)^{(\varphi)}=\left(\eta_{i_{1}} /|\varphi|, \eta_{i_{2}} /|\varphi|, \ldots, \eta_{i_{r}} /|\varphi|\right), \quad \text { with } \quad \theta_{i_{1}}, \theta_{i_{2}}, \ldots, \theta_{i_{r}} \in \varphi .
$$

It follows from a short calculation that if $\boldsymbol{\nu} \in \mathcal{P}^{\Theta}$ has support $\left\{\varphi_{1}, \varphi_{2}, \ldots, \varphi_{r}\right\}$, then the preimage $\boldsymbol{\tau}_{\Theta}^{-1}(\nu)$ has size

$$
\begin{aligned}
& \prod_{j=1}^{r}\left|\varphi_{j}\right|^{\ell\left(\boldsymbol{\nu}^{\left(\varphi_{j}\right)}\right)} \prod_{i \geqslant 1}\left(\begin{array}{c}
m_{i}\left(\boldsymbol{\nu}_{u}^{(\{1\})}\right) \\
m_{i /\left|\varphi_{1}\right|}\left(\boldsymbol{\nu}^{\left(\varphi_{1}\right)}\right), m_{i /\left|\varphi_{2}\right|}\left(\boldsymbol{\nu}^{\left(\varphi_{2}\right)}\right), \cdots, m_{i /\left|\varphi_{r}\right|}\left(\boldsymbol{\nu}^{\left(\varphi_{r}\right)}\right)
\end{array}\right) \\
& =\prod_{\varphi \in \Theta}|\varphi|^{\ell\left(\boldsymbol{\nu}^{(\varphi)}\right)} \prod_{i \geqslant 1} \frac{\left(m_{i}\left(\boldsymbol{\nu}_{u}^{(\{1\})}\right)\right) !}{\prod_{\varphi \in \Theta}\left(m_{i /|\varphi|}\left(\boldsymbol{\nu}^{(\varphi)}\right)\right) !} .
\end{aligned}
$$

The conjugacy classes $K^{\boldsymbol{\mu}}$ of $U_{n}$ are parametrized by $\boldsymbol{\mu} \in \mathcal{P}_{n}^{\Phi}$, a fact on which we elaborate in Section 2.5. We have another natural surjection,

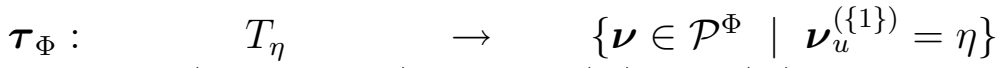

$$
\begin{aligned}
& t=\left(t_{1}, t_{2}, \ldots, t_{\ell}\right) \mapsto \boldsymbol{\tau}_{\Phi}\left(t_{1}\right) \cup \boldsymbol{\tau}_{\Phi}\left(t_{2}\right) \cup \cdots \cup \boldsymbol{\tau}_{\Phi}\left(t_{\ell}\right),
\end{aligned}
$$

where

$$
\boldsymbol{\tau}_{\Phi}\left(t_{i}\right)=\boldsymbol{\mu}^{\prime}, \quad \text { if } t_{i} \in K^{\boldsymbol{\mu}} \text { in } U_{\eta_{i}} .
$$




\subsection{The characteristic map}

For every $f \in \Phi$, let $X^{(f)}=\left\{X_{1}^{(f)}, X_{2}^{(f)}, \ldots\right\}$ be an infinite set of variables, and for every $\varphi \in \Theta$, let $Y^{(\varphi)}=\left\{Y_{1}^{(\varphi)}, Y_{2}^{(\varphi)}, \ldots\right\}$ be an infinite set of variables. We relate symmetric functions in the variables $X^{(f)}$ to those in the variables $Y^{(\varphi)}$ through the transform

$$
p_{k}\left(Y^{(\varphi)}\right)=(-1)^{k|\phi|-1} \sum_{x \in T_{k|\varphi|}} \xi(x) p_{k|\varphi| /\left|f_{x}\right|}\left(X^{\left(f_{x}\right)}\right), \quad \text { where } \xi \in \varphi, x \in f_{x} .
$$

The ring of symmetric functions $\Lambda$ is

$$
\Lambda=\bigotimes_{f \in \Phi} \Lambda\left(X^{(f)}\right)=\bigotimes_{\varphi \in \Theta} \Lambda\left(Y^{(\varphi)}\right)
$$

For $\boldsymbol{\mu} \in \mathcal{P}^{\Phi}$, the Hall-Littlewood polynomial $P_{\boldsymbol{\mu}}$ is

$$
P_{\boldsymbol{\mu}}=(-q)^{-n(\boldsymbol{\mu})} \prod_{f \in \Phi} P_{\boldsymbol{\mu}(f)}\left(X^{(f)} ;(-q)^{-|f|}\right),
$$

and for $\boldsymbol{\lambda} \in \mathcal{P}^{\Theta}$, the power-sum symmetric function $p_{\boldsymbol{\lambda}}$ and the Schur function $s_{\boldsymbol{\lambda}}$ are

$$
p_{\boldsymbol{\lambda}}=\prod_{\varphi \in \Theta} p_{\boldsymbol{\lambda}^{(\varphi)}}\left(Y^{(\varphi)}\right) \quad \text { and } \quad s_{\boldsymbol{\lambda}}=\prod_{\varphi \in \Theta} s_{\boldsymbol{\lambda}^{(\varphi)}}\left(Y^{(\varphi)}\right) .
$$

For $\boldsymbol{\mu}, \boldsymbol{\nu} \in \mathcal{P}^{\Phi}$, the Green function is

$$
Q_{\boldsymbol{\nu}}^{\boldsymbol{\mu}}(-q)=\prod_{f \in \Phi_{\boldsymbol{\mu}}} Q_{\boldsymbol{\nu}^{(f)}}^{\boldsymbol{\mu}^{(f)}}\left((-q)^{|f|}\right),
$$

where $\Phi_{\boldsymbol{\mu}}=\left\{f \in \Phi \mid \boldsymbol{\mu}^{(f)} \neq \emptyset\right\}$. As a graded rings,

$$
\begin{aligned}
\Lambda & =\mathbb{C}-\operatorname{span}\left\{p_{\boldsymbol{\nu}} \mid \boldsymbol{\nu} \in \mathcal{P}^{\Theta}\right\} \\
& =\mathbb{C}-\operatorname{span}\left\{s_{\boldsymbol{\lambda}} \mid \boldsymbol{\lambda} \in \mathcal{P}^{\Theta}\right\} \\
& =\mathbb{C}-\operatorname{span}\left\{P_{\boldsymbol{\mu}} \mid \boldsymbol{\mu} \in \mathcal{P}^{\Phi}\right\} .
\end{aligned}
$$

The conjugacy classes $K^{\boldsymbol{\mu}}$ of $U_{n}$ are indexed by $\boldsymbol{\mu} \in \mathcal{P}_{n}^{\Phi}$ and the irreducible characters $\chi^{\boldsymbol{\lambda}}$ of $U_{n}$ are indexed by $\boldsymbol{\lambda} \in \mathcal{P}_{n}^{\Theta}[5,6]$. Thus, the ring of class functions $C_{n}$ of $U_{n}$ is given by

$$
\begin{aligned}
C_{n} & =\mathbb{C}-\operatorname{span}\left\{\chi^{\boldsymbol{\lambda}} \mid \boldsymbol{\lambda} \in \mathcal{P}_{n}^{\Theta}\right\} \\
& =\mathbb{C}-\operatorname{span}\left\{\kappa^{\boldsymbol{\mu}} \mid \boldsymbol{\mu} \in \mathcal{P}_{n}^{\Phi}\right\}
\end{aligned}
$$

where $\kappa^{\mu}: U_{n} \rightarrow \mathbb{C}$ is given by

$$
\kappa^{\boldsymbol{\mu}}(g)= \begin{cases}1 & \text { if } g \in K^{\boldsymbol{\mu}} \\ 0 & \text { otherwise }\end{cases}
$$


We let $\chi^{\boldsymbol{\lambda}}(\boldsymbol{\mu})$ denote the value of the character $\chi^{\boldsymbol{\lambda}}$ on any element in the conjugacy $K^{\boldsymbol{\mu}}$.

For $\boldsymbol{\nu} \in \mathcal{P}_{n}^{\Theta}$, let the Deligne-Lusztig character $R_{\nu}=R_{\nu}^{U_{n}}$ be given by

$$
R_{\boldsymbol{\nu}}=R_{T_{\boldsymbol{\nu}_{u}}}^{U_{n}}(\theta)
$$

where $\theta \in \operatorname{Hom}\left(T_{\boldsymbol{\nu}_{u}}, \mathbb{C}^{\times}\right)$is any homomorphism such that $\boldsymbol{\tau}_{\Theta}(\theta)=\boldsymbol{\nu}$ (see $(2.12)$ ).

Let $C=\bigoplus_{n \geqslant 1} C_{n}$ so that

$$
\begin{aligned}
C & =\mathbb{C}-\operatorname{span}\left\{\chi^{\boldsymbol{\lambda}} \mid \boldsymbol{\lambda} \in \mathcal{P}^{\Theta}\right\} \\
& =\mathbb{C}-\operatorname{span}\left\{\kappa^{\boldsymbol{\mu}} \mid \boldsymbol{\mu} \in \mathcal{P}^{\Phi}\right\} \\
& =\mathbb{C}-\operatorname{span}\left\{R_{\boldsymbol{\nu}} \quad \mid \boldsymbol{\nu} \in \mathcal{P}^{\Theta}\right\}
\end{aligned}
$$

is a ring with multiplication given by

$$
R_{\boldsymbol{\lambda}} R_{\boldsymbol{\eta}}=R_{\boldsymbol{\lambda} \cup \boldsymbol{\eta}}
$$

The next theorem follows from the results of $[4,6,8,10,16,23]$. A summary of the relevant results in these papers and how they imply the following theorem is given in [23].

Theorem 2.1 (Characteristic Map). The map

$$
\begin{aligned}
& \text { ch }: C \rightarrow \quad \Lambda \\
& \chi^{\boldsymbol{\lambda}} \mapsto(-1)^{\lfloor|\boldsymbol{\lambda}| / 2\rfloor+n(\boldsymbol{\lambda})} s_{\boldsymbol{\lambda}} \\
& \kappa^{\mu} \mapsto \quad P_{\mu} \\
& R_{\boldsymbol{\nu}} \mapsto \quad(-1)^{|\boldsymbol{\nu}|-\ell(\boldsymbol{\nu})} p_{\boldsymbol{\nu}}
\end{aligned}
$$

is an isometric ring isomorphism with respect to the natural inner products

$$
\left\langle\chi^{\boldsymbol{\lambda}}, \chi^{\boldsymbol{\eta}}\right\rangle=\delta_{\boldsymbol{\lambda} \boldsymbol{\eta}} \quad \text { and } \quad\left\langle s_{\boldsymbol{\lambda}}, s_{\boldsymbol{\eta}}\right\rangle=\delta_{\boldsymbol{\lambda} \boldsymbol{\eta}}
$$

In the following change of basis equations, (2.15) follows from Theorem 2.1, (2.16) follows from (2.1), and (2.17) follows from [23, Theorem 4.2].

$$
\begin{array}{rlr}
(-1)^{\lfloor k / 2\rfloor+n(\boldsymbol{\lambda})} s_{\boldsymbol{\lambda}} & =\sum_{\boldsymbol{\mu} \in \mathcal{P}_{k}^{\Phi}} \chi^{\boldsymbol{\lambda}}(\boldsymbol{\mu}) P_{\boldsymbol{\mu}} & \text { for } \boldsymbol{\lambda} \in \mathcal{P}_{k}^{\Theta}, \\
s_{\boldsymbol{\lambda}} & =\sum_{\substack{\boldsymbol{\nu} \in \mathcal{P}_{k}^{\Theta} \\
\boldsymbol{\lambda}_{s}=\boldsymbol{\nu}_{s}}}\left(\prod_{\varphi \in \Theta} \frac{\omega^{\boldsymbol{\lambda}^{(\varphi)}}\left(\boldsymbol{\nu}^{(\varphi)}\right)}{\left.z_{\boldsymbol{\nu}^{(\varphi)}}\right) p_{\boldsymbol{\nu}}}\right. & \text { for } \boldsymbol{\lambda} \in \mathcal{P}_{k}^{\Theta}, \\
(-1)^{k-\ell(\boldsymbol{\nu})} p_{\boldsymbol{\nu}}= & \sum_{\boldsymbol{\mu} \in \mathcal{P}_{k}^{\Phi}}\left(\sum_{\substack{t \in T_{\boldsymbol{\nu}_{u}} \\
\boldsymbol{\tau}_{\Phi}(t)_{s}=\boldsymbol{\mu}_{s}}} \theta(t) Q_{\boldsymbol{\tau}_{\Phi}(t)}^{\boldsymbol{\mu}}(-q)\right) P_{\boldsymbol{\mu}} & \text { for } \boldsymbol{\nu} \in \mathcal{P}_{k}^{\Theta}, \boldsymbol{\tau}_{\Theta}(\theta)=\boldsymbol{\nu}
\end{array}
$$




\section{Gelfand-Graev characters on arbitrary elements}

\section{1 $G_{n}=\operatorname{GL}\left(n, \mathbb{F}_{q}\right)$ notation}

In this Section 3, let

$$
\tilde{\Phi}=\left\{\tilde{F} \text {-orbits in } \overline{\mathbb{F}}_{q}^{\times}\right\} .
$$

Define norm maps $\tilde{N}_{m, k}: \tilde{T}_{(m)} \rightarrow \tilde{T}_{(k)}$, whenever $k \mid m$, the same as in (2.8), except by replacing $-q$ by $q$, and define the corresponding transpose maps $\tilde{N}_{m, k}^{*}: \tilde{T}_{(m)}^{*} \rightarrow \tilde{T}_{(k)}^{*}$ as in (2.9), where $\tilde{T}_{(m)}^{*}$ is the character group of $\tilde{T}_{(m)}$. We now let $\tilde{L}$ be the direct limit of the groups $\tilde{T}_{(m)}$ with respect to the maps $\tilde{N}_{m, k}^{*}$ :

$$
\tilde{L}=\underset{\lim }{\longrightarrow} \tilde{T}_{(m)},
$$

and since $\tilde{F}$ acts on $\tilde{L}$, we may consider the corresponding orbits, and we define

$$
\tilde{\Theta}=\{\tilde{F} \text {-orbits in } \tilde{L}\} \text {. }
$$

The same set-up of Sections 2.4 and 2.5 gives a characteristic map for $G_{n}=\operatorname{GL}\left(n, \mathbb{F}_{q}\right)$ by replacing $\Phi$ by $\tilde{\Phi}, \Theta$ by $\tilde{\Theta},-q$ by $q, T_{(k)}$ by $\tilde{T}_{(k)}$, and $(-1)^{\lfloor n / 2\rfloor+n(\boldsymbol{\lambda})} s_{\boldsymbol{\lambda}}$ by $s_{\boldsymbol{\lambda}}$. With the exception of the Deligne-Lusztig characters (which follows from the parallel argument of [23, Theorem 4.2]), this can be found in [17, Chapter IV].

\subsection{The Gelfand-Graev character}

We will use $U_{n}^{\prime}=\mathrm{GL}\left(n, \overline{\mathbb{F}}_{q}\right)^{F^{\prime}}$ (see (2.7)) to give an explicit description of the GelfandGraev character. For a more general description see [4], for example.

For $1 \leqslant i<j \leqslant n$ and $t \in \mathbb{F}_{q}$, let $x_{i j}(t)$ denote the matrix with ones on the diagonal, $t$ in the $i$ th row and $j$ th column, and zeroes elsewhere. Let

$$
\begin{aligned}
u_{i j}(t) & =x_{i j}(t) x_{n+1-j, n+1-i}\left(-t^{q}\right) & & \text { for } 1 \leqslant i<j \leqslant\lfloor n / 2\rfloor, t \in \mathbb{F}_{q^{2}}, \\
u_{i, n+1-j}(t) & =x_{i, n+1-j}(t) x_{j, n+1-i}\left(-t^{q}\right) & & \text { for } 1 \leqslant i<j \leqslant\lfloor n / 2\rfloor, t \in \mathbb{F}_{q^{2}},
\end{aligned}
$$

and for $1 \leqslant k \leqslant\lfloor n / 2\rfloor$, and $t, a, b \in \mathbb{F}_{q^{2}}$, let

$$
\begin{aligned}
u_{k}(a) & =x_{k, n+1-k}(a) & & \text { for } n \text { even, and } a^{q}+a=0, \\
u_{k}(a, b) & =x_{\lceil n / 2\rceil, n+1-k}\left(-a^{q}\right) x_{k, n+1-k}(b) x_{k,\lceil n / 2\rceil}(a) & & \text { for } n \text { odd, and } a^{q+1}+b+b^{q}=0 .
\end{aligned}
$$

Examples. In $U_{4}^{\prime}$, we have

$$
u_{12}(t)=\left(\begin{array}{cccc}
1 & t & 0 & 0 \\
0 & 1 & 0 & 0 \\
0 & 0 & 1 & -t^{q} \\
0 & 0 & 0 & 1
\end{array}\right), \quad u_{13}(t)=\left(\begin{array}{cccc}
1 & 0 & t & 0 \\
0 & 1 & 0 & -t^{q} \\
0 & 0 & 1 & 0 \\
0 & 0 & 0 & 1
\end{array}\right), \quad u_{1}(a)=\left(\begin{array}{cccc}
1 & 0 & 0 & a \\
0 & 1 & 0 & 0 \\
0 & 0 & 1 & 0 \\
0 & 0 & 0 & 1
\end{array}\right), \quad u_{2}(a)=\left(\begin{array}{cccc}
1 & 0 & 0 & 0 \\
0 & 1 & a & 0 \\
0 & 0 & 1 & 0 \\
0 & 0 & 0 & 1
\end{array}\right),
$$

where $a^{q}+a=0$. In $U_{5}^{\prime}$, we have

$$
u_{12}(t)=\left(\begin{array}{ccccc}
1 & t & 0 & 0 & 0 \\
0 & 1 & 0 & 0 & 0 \\
0 & 0 & 0 & 0 & 0 \\
0 & 0 & 0 & 1 & -t^{q} \\
0 & 0 & 0 & 0 & 1
\end{array}\right), \quad u_{14}(t)=\left(\begin{array}{ccccc}
1 & 0 & 0 & t & 0 \\
0 & 1 & 0 & 0 & -t^{q} \\
0 & 0 & 1 & 0 & 0 \\
0 & 0 & 0 & 1 & 0 \\
0 & 0 & 0 & 0 & 1
\end{array}\right),
$$




$$
u_{1}(a, b)=\left(\begin{array}{ccccc}
1 & 0 & a & 0 & b \\
0 & 1 & 0 & 0 & 0 \\
0 & 0 & 1 & 0 & -a^{q} \\
0 & 0 & 0 & 1 \\
0 & 0 & 0 & 0 & 0
\end{array}\right), \quad u_{2}(a, b)=\left(\begin{array}{ccccc}
1 & 0 & 0 & 0 & 0 \\
0 & 1 & a & b & 0 \\
0 & 0 & 1 & -a^{q} & 0 \\
0 & 0 & 0 & 1 \\
0 & 0 & 0 & 0 & 0 \\
0 & 0 & 0
\end{array}\right)
$$

where $a^{q+1}+b+b^{q}=0$.

For $1 \leqslant i<j \leqslant\lfloor n / 2\rfloor$ and $1 \leqslant k \leqslant\lfloor n / 2\rfloor$, define the one-parameter subgroups

$$
\begin{aligned}
\mathfrak{X}_{i j} & =\left\{u_{i j}(t) \mid t \in \mathbb{F}_{q^{2}}\right\} \cong \mathbb{F}_{q^{2}}^{+}, \\
\mathfrak{X}_{i, n+1-j} & =\left\{u_{i, n+1-j}(t) \mid t \in \mathbb{F}_{q^{2}}\right\} \cong \mathbb{F}_{q^{2}}^{+}, \\
\mathfrak{X}_{k} & = \begin{cases}\left\{u_{k}(t) \mid t \in \mathbb{F}_{q^{2}}, t^{q}+t=0\right\} & \text { if } n \text { is even, } \\
\left\{u_{k}(a, b) \mid a, b \in \mathbb{F}_{q^{2}}, a^{q+1}+b+b^{q}=0\right\} & \text { if } n \text { is odd }\end{cases}
\end{aligned}
$$

so that

$$
B_{n}^{<}=\left\langle\mathfrak{X}_{i j}, \mathfrak{X}_{i, n-j}, \mathfrak{X}_{k} \mid 1 \leqslant i<j \leqslant\lfloor n / 2\rfloor, 1 \leqslant k \leqslant\lfloor n / 2\rfloor\right\rangle \subseteq U_{n}^{\prime}
$$

is the subgroup of $U_{n}^{\prime}$ of upper-triangular matrices with ones on the diagonal. Noting that

$$
\mathfrak{X}_{k} /\left[\mathfrak{X}_{k}, \mathfrak{X}_{k}\right] \cong \begin{cases}\mathbb{F}_{q}^{+} & \text {if } n \text { is even } \\ \mathbb{F}_{q^{2}}^{+} & \text {if } n \text { is odd }\end{cases}
$$

a direct calculation gives

$$
\begin{aligned}
B_{n}^{<} /\left[B_{n}^{<}, B_{n}^{<}\right] & \cong \mathfrak{X}_{12} \times \mathfrak{X}_{23} \times \ldots \times \mathfrak{X}_{\lfloor n / 2\rfloor-1,\lfloor n / 2\rfloor} \times \mathfrak{X}_{\lfloor n / 2\rfloor} \\
& \cong \begin{cases}\left(\mathbb{F}_{q^{2}}^{+}\right)^{(n / 2)-1} \times \mathbb{F}_{q}^{+} & \text {if } n \text { is even }, \\
\left(\mathbb{F}_{q^{2}}^{+}\right)^{\lfloor n / 2\rfloor} & \text { if } n \text { is odd } .\end{cases}
\end{aligned}
$$

Similarly, let

$$
\tilde{B}_{n}^{<}=\left\langle x_{i j}(t) \mid 1 \leqslant i<j \leqslant n, t \in \mathbb{F}_{q}\right\rangle \subseteq G_{n}
$$

be the subgroup of unipotent upper-triangular matrices in $G_{n}$.

Fix a homomorphism $\psi: \mathbb{F}_{q^{2}}^{+} \rightarrow \mathbb{C}^{\times}$of the additive group of the field such that for all $1 \leqslant k \leqslant\lfloor n / 2\rfloor, \psi$ is nontrivial on $\mathfrak{X}_{k} /\left[\mathfrak{X}_{k}, \mathfrak{X}_{k}\right]$. Define the homomorphism $\psi_{(n)}: B_{n}^{<} \rightarrow \mathbb{C}$ by

$$
\left.\psi_{(n)}\right|_{\mathfrak{X}_{\alpha} /\left[\mathfrak{X}_{\alpha}, \mathfrak{X}_{\alpha}\right]}= \begin{cases}\psi & \text { if } \alpha=(i, i+1), 1 \leqslant i<\lfloor n / 2\rfloor, \text { or if } \alpha=\lfloor n / 2\rfloor, \\ 1 & \text { otherwise. }\end{cases}
$$

The Gelfand-Graev character of $U_{n}^{\prime}$ is

$$
\Gamma_{n}^{\prime}=\operatorname{Ind}_{B_{n}^{<}}^{U_{n}^{\prime}}\left(\psi_{(n)}\right)
$$

Recall that $U_{n}^{\prime}$ is conjugate to $U_{n}$ in $\bar{G}_{n}$. If $U_{n}^{\prime}=y U_{n} y^{-1}$, then let

$$
\Gamma_{n}=\operatorname{Ind}_{y^{-1} B_{n}^{<} y}^{U_{n}}\left(y^{-1} \psi_{(n)} y\right) .
$$

Similarly, the Gelfand-Graev character $\tilde{\Gamma}_{(n)}$ of $G_{n}$ is

$$
\tilde{\Gamma}_{(n)}=\operatorname{Ind}_{\tilde{B}_{n}^{<}}^{G_{n}}\left(\tilde{\psi}_{(n)}\right)
$$


where $\tilde{\psi}_{(n)}: \tilde{B}_{n}^{<} \rightarrow \mathbb{C}$ is given by

$$
\tilde{\psi}_{(n)}\left(x_{i j}(t)\right)= \begin{cases}\psi(t) & \text { if } j=i+1 \\ 1 & \text { otherwise }\end{cases}
$$

It is well-known that the Gelfand-Graev character has a multiplicity free decomposition into irreducible characters $[22,25,26]$. The following explicit decompositions essentially follow from [3]. Specific proofs are given in [27] in the $G_{n}$ case and in [18] in the $U_{n}$ case.

Theorem 3.1. Let $h t(\boldsymbol{\lambda})=\max \left\{\ell\left(\boldsymbol{\lambda}^{(\varphi)}\right)\right\}$. Then

$$
\Gamma_{(n)}=\sum_{\substack{\boldsymbol{\lambda} \in \mathcal{P}^{\Theta} \\ \operatorname{ht}(\boldsymbol{\lambda})=1}} \chi^{\boldsymbol{\lambda}} \quad \text { and } \quad \tilde{\Gamma}_{(n)}=\sum_{\substack{\boldsymbol{\lambda} \in \mathcal{P}^{\tilde{\Theta}} \\ \operatorname{ht}(\boldsymbol{\lambda})=1}} \chi^{\boldsymbol{\lambda}}
$$

\subsection{The character values of the Gelfand-Graev character}

A unipotent conjugacy class $K^{\boldsymbol{\mu}}$ of $U_{n}$ or $G_{n}$ is a conjugacy class that satisfies

$$
\boldsymbol{\mu}_{u}=\boldsymbol{\mu}
$$

The unipotent conjugacy classes of $U_{n}$ and $G_{n}$ are thus parametrized by partitions $\mu$ of $n$.

\section{Lemma 3.1.}

(a) Let $\boldsymbol{\mu} \in \mathcal{P}_{n}^{\Theta}, \boldsymbol{\mu}_{u}^{(\{1\})}=\mu$. Then

$$
\Gamma_{(n)}(\boldsymbol{\mu})= \begin{cases}\sum_{\nu \in \mathcal{P}_{n}} \frac{(-1)^{n+\lfloor n / 2\rfloor-\ell(\nu)}}{z_{\nu}}\left|T_{\nu}\right| Q_{\nu}^{\mu}(-q) & \text { if } \boldsymbol{\mu} \text { is unipotent } \\ 0 & \text { otherwise. }\end{cases}
$$

(b) Let $\boldsymbol{\mu} \in \mathcal{P}_{n}^{\tilde{\Theta}}, \boldsymbol{\mu}_{u}^{(\{1\})}=\mu$. Then

$$
\tilde{\Gamma}_{(n)}(\boldsymbol{\mu})= \begin{cases}\sum_{\nu \in \mathcal{P}_{n}} \frac{(-1)^{n-\ell(\nu)}}{z_{\nu}}\left|\tilde{T}_{\nu}\right| Q_{\nu}^{\mu}(q) & \text { if } \boldsymbol{\mu} \text { is unipotent } \\ 0 & \text { otherwise. }\end{cases}
$$

Proof. Note that if $h t(\boldsymbol{\lambda}) \leqslant 1$, then $n(\boldsymbol{\lambda})=0$. Thus, by applying the characteristic map and (2.16) to Theorem 3.1,

$$
\operatorname{ch}\left(\Gamma_{(n)}\right)=(-1)^{\lfloor n / 2\rfloor} \sum_{\substack{\boldsymbol{\lambda} \in \mathcal{P}_{n}^{\Theta} \\ \operatorname{ht}(\boldsymbol{\lambda}) \leqslant 1}} \sum_{\substack{\boldsymbol{\nu} \in \mathcal{P}_{n}^{\Theta} \\ \boldsymbol{\nu}_{s}=\boldsymbol{\lambda}_{s}}}\left(\prod_{\varphi \in \Theta} \frac{\omega^{\boldsymbol{\lambda}^{(\varphi)}}\left(\boldsymbol{\nu}^{(\varphi)}\right)}{z_{\boldsymbol{\nu}^{(\varphi)}}}\right) p_{\boldsymbol{\nu}}
$$


Since $h t(\boldsymbol{\lambda}) \leqslant 1, \omega^{\boldsymbol{\lambda}^{(\varphi)}}$ is the trivial character for all $\varphi \in \Theta$. Thus, the summand is independent of $\boldsymbol{\lambda}$, and

$$
\operatorname{ch}\left(\Gamma_{(n)}\right)=(-1)^{\lfloor n / 2\rfloor} \sum_{\boldsymbol{\nu} \in \mathcal{P}_{n}^{\Theta}}\left(\prod_{\varphi \in \Theta} z_{\boldsymbol{\nu}^{(\varphi)}}^{-1}\right) p_{\boldsymbol{\nu}}
$$

By (2.13),

$$
\begin{aligned}
& \operatorname{ch}\left(\Gamma_{(n)}\right) \\
& =(-1)^{\lfloor n / 2\rfloor} \sum_{\boldsymbol{\nu} \in \mathcal{P}_{n}^{\Theta}}\left(\prod_{\varphi \in \Theta}|\varphi|^{\ell\left(\boldsymbol{\nu}^{(\varphi)}\right)} \prod_{i \geqslant 1} \frac{\left(m_{i}\left(\boldsymbol{\nu}_{u}^{(\{1\})}\right)\right) !}{\prod_{\varphi \in \Theta}\left(m_{i /|\varphi|}\left(\boldsymbol{\nu}^{(\varphi)}\right)\right) !}\right)^{-1} \sum_{\substack{\theta \in \operatorname{Hom}_{\boldsymbol{\Theta}}\left(T_{\boldsymbol{\nu} u}, \mathbb{C}^{\times}\right) \\
\boldsymbol{\tau}_{\Theta}(\theta)=\boldsymbol{\nu}}}\left(\prod_{\substack{\boldsymbol{\varphi} \in \Theta \\
z^{(\varphi)}}}^{-1} p_{\boldsymbol{\nu}}\right. \\
& =(-1)^{\lfloor n / 2\rfloor} \sum_{\boldsymbol{\nu} \in \mathcal{P}_{n}^{\Theta}} \sum_{\substack{\theta \in \operatorname{Hom}\left(T_{\boldsymbol{\nu}}, \mathbb{C}^{\times}\right) \\
\boldsymbol{\tau}_{\Theta}(\theta)=\boldsymbol{\nu}}} z_{\boldsymbol{\nu}_{u}}^{-1} p_{\boldsymbol{\nu}} \\
& =(-1)^{\lfloor n / 2\rfloor} \sum_{\boldsymbol{\nu} \in \mathcal{P}_{n}} \sum_{\theta \in \operatorname{Hom}\left(T_{\nu}, \mathbb{C}^{\times}\right)} z_{\nu}^{-1} p_{\boldsymbol{\tau}_{\Theta}(\theta)} .
\end{aligned}
$$

The change of basis (2.17) gives

$$
\begin{aligned}
& \operatorname{ch}\left(\Gamma_{(n)}\right)=(-1)^{\lfloor n / 2\rfloor} \sum_{\nu \in \mathcal{P}_{n}} \sum_{\theta \in \operatorname{Hom}\left(T_{\nu}, \mathbb{C}^{\times}\right)} \frac{(-1)^{n-\ell(\nu)}}{z_{\nu}} \sum_{\boldsymbol{\mu} \in \mathcal{P}_{n}^{\Phi}} \sum_{\substack{t \in T_{\nu} \\
\boldsymbol{\tau}_{\Phi}(t)_{s}=\boldsymbol{\mu}_{s}}} \theta(t) Q_{\boldsymbol{\tau}_{\Phi}(t)}^{\boldsymbol{\mu}}(-q) P_{\boldsymbol{\mu}} \\
& =(-1)^{\lfloor n / 2\rfloor} \sum_{\boldsymbol{\mu} \in \mathcal{P}_{n}^{\Phi}} \sum_{\nu \in \mathcal{P}_{n}} \frac{(-1)^{n-\ell(\nu)}}{z_{\nu}} \sum_{\substack{t \in T_{\nu} \\
\boldsymbol{\tau}_{\Phi}(t)_{s}=\boldsymbol{\mu}_{s}}} \sum_{\theta \in \operatorname{Hom}\left(T_{\nu}, \mathbb{C}^{\times}\right)} \theta(t) Q_{\boldsymbol{\tau}_{\Phi}(t)}^{\boldsymbol{\mu}}(-q) P_{\boldsymbol{\mu}} .
\end{aligned}
$$

By the orthogonality of characters of $T_{\nu}$, the inner-most sum is equal to zero for all $t \neq 1$. If $t=1$, then $\boldsymbol{\tau}_{\Phi}(1,1, \ldots, 1)^{(f)}=\emptyset$ for $f \neq\{1\}$ and $\boldsymbol{\tau}_{\Phi}(1,1, \ldots, 1)^{(\{1\})}=\nu$. Thus,

$$
\operatorname{ch}\left(\Gamma_{(n)}\right)=(-1)^{\lfloor n / 2\rfloor} \sum_{\substack{\boldsymbol{\mu} \in \mathcal{P}_{n}^{\Phi} \\ \boldsymbol{\mu}_{u}=\boldsymbol{\mu}}} \sum_{\nu \in \mathcal{P}_{n}} \frac{(-1)^{n-\ell(\nu)}}{z_{\nu}}\left|T_{\nu}\right| Q_{\nu}^{\boldsymbol{\mu}_{u}}(-q) P_{\boldsymbol{\mu}},
$$

and in particular, if $\boldsymbol{\mu}_{u}^{(\{1\})}=\mu$,

$$
\Gamma_{(n)}(\boldsymbol{\mu})= \begin{cases}\sum_{\nu \in \mathcal{P}_{n}} \frac{(-1)^{n+\lfloor n / 2\rfloor-\ell(\nu)}}{z_{\nu}}\left|T_{\nu}\right| Q_{\nu}^{\mu}(-q) & \text { if } \boldsymbol{\mu} \text { is unipotent } \\ 0 & \text { otherwise }\end{cases}
$$

(b) The proof is similar to (a), just using the $G_{n}$ characteristic map.

Remark. In the proof of Lemma 3.1, one may skip to (3.1) by using 10.7.3 in [3].

The values of the Gelfand-Graev character of the finite general linear group are wellknown. An elementary proof of the following theorem is given in [9]. 
Theorem 3.2. Let $\boldsymbol{\mu} \in \mathcal{P}_{n}^{\tilde{\Phi}}$ with $\mu=\boldsymbol{\mu}_{u}^{(\{1\})}$. Then

$$
\tilde{\Gamma}_{(n)}(\boldsymbol{\mu})= \begin{cases}(-1)^{n-\ell(\mu)} \prod_{i=1}^{\ell(\mu)}\left(q^{i}-1\right) & \text { if } \boldsymbol{\mu} \text { is unipotent }, \\ 0 & \text { otherwise. }\end{cases}
$$

We may now apply Theorem 3.2 and Lemma 3.1 to give the values of the GelfandGraev character of $U_{n}$.

Corollary 3.1. Let $\boldsymbol{\mu} \in \mathcal{P}_{n}^{\Phi}$ with $\mu=\boldsymbol{\mu}_{u}^{(\{1\})}$. Then

$$
\Gamma_{(n)}(\boldsymbol{\mu})= \begin{cases}(-1)^{\lfloor n / 2\rfloor-\ell(\mu)} \prod_{i=1}^{\ell(\mu)}\left((-q)^{i}-1\right) & \text { if } \boldsymbol{\mu} \text { is unipotent } \\ 0 & \text { otherwise. }\end{cases}
$$

Proof. Combine Lemma 3.1 (b) with Theorem 3.2 to get

$$
(-1)^{\ell(\mu)} \prod_{i=1}^{\ell(\mu)}\left(q^{i}-1\right)=\sum_{\nu \in \mathcal{P}_{n}} \frac{(-1)^{\ell(\nu)}}{z_{\nu}}\left|\tilde{T}_{\nu}\right| Q_{\nu}^{\mu}(q)
$$

which implies

$$
\prod_{i=1}^{\ell(\mu)}\left(1-q^{i}\right)=\sum_{\nu \in \mathcal{P}_{n}} \frac{1}{z_{\nu}} \prod_{i=1}^{\ell(\nu)}\left(1-q^{\nu_{i}}\right) Q_{\nu}^{\mu}(q) .
$$

Make the substitution $q \mapsto-q$ to get

$$
\prod_{i=1}^{\ell(\mu)}\left(1-(-q)^{i}\right)=\sum_{\nu \in \mathcal{P}_{n}} \frac{1}{z_{\nu}} \prod_{i=1}^{\ell(\nu)}\left(1-(-q)^{\nu_{i}}\right) Q_{\nu}^{\mu}(-q)
$$

which implies

$$
(-1)^{\lfloor n / 2\rfloor+\ell(\mu)} \prod_{i=1}^{\ell(\mu)}\left((-q)^{i}-1\right)=\sum_{\nu \in \mathcal{P}_{n}} \frac{(-1)^{\lfloor n / 2\rfloor+|\nu|-\ell(\nu)}}{z_{\nu}}\left|T_{\nu}\right| Q_{\nu}^{\mu}(-q) .
$$

Apply this last identity to Lemma 3.1 (a) to obtain the desired result.

\section{Degenerate Gelfand-Graev characters}

\section{1 $G_{n}=\mathrm{GL}\left(n, \mathbb{F}_{q^{2}}\right)$ notation (different from Section 3)}

In this Section 4 , let $G_{n}=\operatorname{GL}\left(n, \mathbb{F}_{q^{2}}\right)$, and define

$$
\tilde{\Phi}=\left\{F^{2} \text {-orbits of } \overline{\mathbb{F}}_{q}^{\times}\right\} .
$$

Note that now $G_{n}^{F}=U_{n}$ and $G_{n}^{F^{\prime}}=U_{n}^{\prime}$, and also that $\tilde{T}_{2 m}=T_{2 m}$. Through the norm maps $\tilde{N}_{2 m, 2 k}: T_{(2 m)} \rightarrow T_{(2 k)}$ (where $k \mid m$ ), defined in (2.8), and the corresponding transpose 
maps $\tilde{N}_{2 m, 2 k}^{*}: T_{(2 m)}^{*} \rightarrow T_{(2 k)}^{*}$ defined in (2.9), we let $\tilde{L}$ be the direct limit of the groups $T_{(2 m)}^{*}$ with respect to the maps $N_{2 m, 2 k}^{*}$ :

$$
\tilde{L}=\underset{\lim }{\longrightarrow} T_{(2 m)}^{*} .
$$

Since $F^{2}=\tilde{F}^{2}$ acts on $\tilde{L}$, we may consider the corresponding orbits, and define

$$
\tilde{\Theta}=\left\{F^{2} \text {-orbits in } \tilde{L}\right\} \text {. }
$$

The same set-up of Sections 2.4 and 2.5 gives a characteristic map for $G_{n}$ by replacing $\Phi$ by $\tilde{\Phi}, \Theta$ by $\tilde{\Theta},-q$ by $q, T_{(k)}$ by $T_{(2 k)}$, and $(-1)^{\lfloor n / 2\rfloor+n(\lambda)} s_{\boldsymbol{\lambda}}$ by $s_{\boldsymbol{\lambda}}$.

\subsection{The definition of degenerate Gelfand-Graev characters}

Let $(k, \nu)$ be a pair such that $\nu \vdash \frac{n-k}{2} \in \mathbb{Z}_{\geqslant 0}$, and let

$$
\nu_{\leqslant}=\left(\nu_{\leqslant 1}, \nu_{\leqslant 2}, \ldots, \nu_{\leqslant \ell}\right), \quad \text { where } \quad \nu_{\leqslant j}=\nu_{1}+\nu_{2}+\cdots+\nu_{j} .
$$

Then the map $\psi_{(k, \nu)}: B_{n}^{<} \rightarrow \mathbb{C}^{\times}$, given by

$$
\left.\psi_{(k, \nu)}\right|_{\mathfrak{X}_{\alpha} /\left[\mathfrak{x}_{\alpha}, \mathfrak{X}_{\alpha}\right]}= \begin{cases}\psi & \text { if } \alpha=(i, i+1), 1 \leqslant i<\lfloor n / 2\rfloor, \text { and } i \notin \nu \leqslant, \\ \psi & \text { if } \alpha=\lfloor n / 2\rfloor \text { and }\lfloor n / 2\rfloor \notin \nu_{\leqslant}, \\ 1 & \text { otherwise, }\end{cases}
$$

is a linear character of $U_{n}^{\prime}$. Note that $\psi_{\left([n / 2\rceil-\lfloor n / 2\rfloor,\left(1^{\lfloor n / 2\rfloor}\right)\right)}$ is the trivial character and $\psi_{(n, \emptyset)}=\psi_{(n)}$ of Section 3 .

The degenerate Gelfand-Graev character $\Gamma_{(k, \nu)}$ is

$$
\Gamma_{(k, \nu)}=\operatorname{Ind}_{B_{n}^{<}}^{U_{n}^{\prime}}\left(\psi_{(k, \nu)}\right) \cong \operatorname{Ind}_{y B_{n}^{<} y^{-1}}^{U_{n}}\left(y \psi_{(k, \nu)} y^{-1}\right),
$$

where $y$ is an element of $\bar{G}_{n}$ such that $y U_{n}^{\prime} y^{-1}=U_{n}$. In particular, the Gelfand-Graev character is $\Gamma_{(n, \emptyset)}$.

Let

where

$$
L_{(k, \nu)}^{\prime}=\left\langle L_{k}, L_{\nu}^{(1)}, L_{\nu}^{(2)}, \cdots, L_{\nu}^{(\ell)}\right\rangle
$$

$$
\begin{aligned}
L_{k} & =\left\langle\mathfrak{X}_{i j}, \mathfrak{X}_{i, n+1-j}, \mathfrak{X}_{r}|| \nu|<i<j \leqslant| \nu|+k,| \nu|\leqslant r \leqslant| \nu \mid+k\right\rangle \cong \mathrm{U}\left(k, \mathbb{F}_{q^{2}}\right) \\
L_{\nu}^{(r)} & =\left\langle\mathfrak{X}_{i j} \mid \nu_{\leqslant r-1} \leqslant i<j \leqslant \nu_{\leqslant r}\right\rangle \cong \operatorname{GL}\left(\nu_{r}, \mathbb{F}_{q^{2}}\right) .
\end{aligned}
$$

Then

$$
L_{(k, \nu)}^{\prime} \cong \mathrm{U}\left(k, \mathbb{F}_{q^{2}}\right) \oplus \mathrm{GL}\left(\nu_{1}, \mathbb{F}_{q^{2}}\right) \oplus \cdots \oplus \mathrm{GL}\left(\nu_{\ell}, \mathbb{F}_{q^{2}}\right)
$$

is a maximally split Levi subgroup of $U_{n}^{\prime}$. For example, if $n=9, k=3$, and $\nu=(2,1)$, then

$$
L_{(k, \nu)}^{\prime}=\left\{\left(\begin{array}{ccccc}
A & 0 & 0 & 0 & 0 \\
0 & B & 0 & 0 & 0 \\
0 & 0 & C & 0 & 0 \\
0 & 0 & 0 & F^{\prime}(B) & 0 \\
0 & 0 & 0 & 0 & F^{\prime}(A)
\end{array}\right) \mid \begin{array}{l}
A \in \mathrm{GL}\left(2, \mathbb{F}_{q^{2}}\right), B \in \mathrm{GL}\left(1, \mathbb{F}_{q^{2}}\right), \\
C \in \mathrm{U}\left(3, \mathbb{F}_{q^{2}}\right)
\end{array}\right\} .
$$


Note that since $L_{\nu}^{(i)} \subseteq U_{2 \nu_{i}}^{\prime} \cong U_{2 \nu_{i}}$, the Levi subgroup

$$
U_{(k, \nu)}=U_{k} \oplus U_{2 \nu_{1}} \oplus U_{2 \nu_{2}} \oplus \cdots \oplus U_{2 \nu_{\ell}} \subseteq U_{n}
$$

contains a Levi subgroup $L=U_{k} \oplus L_{1} \oplus \cdots \oplus L_{\ell}$ with $L_{i} \subseteq U_{2 \nu_{i}}$ such that $L \cong L_{(k, \nu)}^{\prime}$.

Proposition 4.1. Let $(k, \nu)$ be such that $\nu \vdash \frac{n-k}{2} \in \mathbb{Z}_{\geqslant 0}$. Then

$$
\operatorname{ch}\left(\Gamma_{(k, \nu)}\right)=\operatorname{ch}\left(\Gamma_{(k)}\right) \operatorname{ch}\left(R_{G_{\nu_{1}}}^{U_{2 \nu_{1}}}\left(\tilde{\Gamma}_{\left(\nu_{1}\right)}\right)\right) \operatorname{ch}\left(R_{G_{\nu_{2}}}^{U_{2 \nu_{2}}}\left(\tilde{\Gamma}_{\left(\nu_{2}\right)}\right)\right) \cdots \operatorname{ch}\left(R_{G_{\nu_{\ell}}}^{U_{2 \nu_{\ell}}}\left(\tilde{\Gamma}_{\left(\nu_{\ell}\right)}\right)\right)
$$

where $\tilde{\Gamma}_{(m)}$ is the Gelfand-Graev character of $G_{m}=\operatorname{GL}\left(m, \mathbb{F}_{q^{2}}\right)$.

This proposition is a consequence of Theorem 2.1 and the following lemma.

Lemma 4.1. Let $(k, \nu)$ be such that $\nu \vdash \frac{n-k}{2} \in \mathbb{Z}_{\geqslant 0}$. Then

$$
\Gamma_{(k, \nu)} \cong R_{U_{(k, \nu)}}^{U_{n}}\left(\Gamma_{(k)} \otimes R_{L_{1}}^{U_{2 \nu_{1}}}\left(\tilde{\Gamma}_{\left(\nu_{1}\right)}\right) \otimes \cdots \otimes R_{L_{\ell}}^{U_{2 \nu_{\ell}}}\left(\tilde{\Gamma}_{\left(\nu_{\ell}\right)}\right)\right)
$$

Proof. Since $L_{(k, \nu)}^{\prime}$ is maximally split,

$$
\operatorname{Ind}_{y B_{n}^{<} y^{-1}}^{U_{n}}\left(y \psi_{(k, \nu)} y^{-1}\right) \cong \operatorname{Ind}_{B_{n}^{<}}^{U_{n}^{\prime}}\left(\psi_{(k, \nu)}\right) \cong \operatorname{Indf}_{L_{(k, \nu)}^{\prime}}^{U_{n}^{\prime}}\left(\Gamma_{(k)} \otimes \tilde{\Gamma}_{\left(\nu_{1}\right)} \otimes \cdots \otimes \tilde{\Gamma}_{\left(\nu_{\ell}\right)}\right) .
$$

where $\operatorname{Indf}_{L}^{G}$ is Harish-Chandra induction. However,

$$
\begin{aligned}
& \operatorname{Indf}_{L_{(k, \nu)}^{\prime}}^{U_{n}^{\prime}}\left(\Gamma_{(k)} \otimes \tilde{\Gamma}_{\left(\nu_{1}\right)} \otimes \cdots \otimes \tilde{\Gamma}_{\left(\nu_{\ell}\right)}\right)=R_{L_{(k, \nu)}^{U_{n}^{\prime}}}^{U^{\prime}}\left(\Gamma_{(k)} \otimes \tilde{\Gamma}_{\left(\nu_{1}\right)} \otimes \cdots \otimes \tilde{\Gamma}_{\left(\nu_{\ell}\right)}\right), \\
& \quad \cong R_{L}^{U_{n}}\left(\Gamma_{(k)} \otimes \tilde{\Gamma}_{\left(\nu_{1}\right)} \otimes \cdots \otimes \tilde{\Gamma}_{\left(\nu_{\ell}\right)}\right) .
\end{aligned}
$$

By transitivity of Deligne-Lusztig induction, we now have

$$
\operatorname{Ind}_{y B_{n}^{<} y^{-1}}^{U_{n}}\left(y \psi_{(k, \nu)} y^{-1}\right) \cong R_{U_{(k, \nu)}}^{U_{n}}\left(\Gamma_{(k)} \otimes R_{L_{1}}^{U_{2 \nu_{1}}}\left(\tilde{\Gamma}_{\left(\nu_{1}\right)}\right) \otimes \cdots \otimes R_{L_{\ell}}^{U_{2 \nu_{\ell}}}\left(\tilde{\Gamma}_{\left(\nu_{\ell}\right)}\right)\right) .
$$

\subsection{Symplectic tableaux and domino tableaux combinatorics}

Augment the nonnegative integers by symbols $\left\{\bar{i} \mid i \in \mathbb{Z}_{>0}\right\}$, so that we have

$$
\{0, \overline{1}, 1, \overline{2}, 2, \overline{3}, 3, \ldots\}
$$

and order this set by $i-1<\bar{i}<i<\overline{i+1}$. Alternatively, one could identify this augmented set with $\frac{1}{2} \mathbb{Z}_{\geqslant 0}$ by $\bar{i}=i-\frac{1}{2}$.

Let $\lambda=\left(\lambda_{1}, \lambda_{2}, \ldots, \lambda_{r}\right)$ be a partition of $n$ and $\left(m_{0}, m_{1}, m_{2}, \ldots, m_{\ell}\right)$ be a sequence of nonnegative integers that sum to $n$ with $m_{0} \leqslant \lambda_{1}$. A symplectic tableau $Q$ of shape $\lambda /\left(m_{0}\right)$ and weight $\left(m_{0}, m_{1}, \ldots, m_{\ell}\right)$ is a column strict filling of the boxes of $\lambda$ by symbols

$$
\{0, \overline{1}, 1, \overline{2}, 2, \ldots, \bar{\ell}, \ell\}
$$


such that

$$
m_{i}= \begin{cases}\text { number of } 0 \text { 's in } Q & \text { if } i=0, \\ \text { number of } \bar{i} \text { 's }+ \text { number of } i \text { 's in } Q & \text { if } i>0 .\end{cases}
$$

We write $\operatorname{sh}(Q)=\lambda /\left(m_{0}\right)$ and $\operatorname{wt}(Q)=\left(m_{0}, m_{1}, \ldots, m_{\ell}\right)$. For example, if

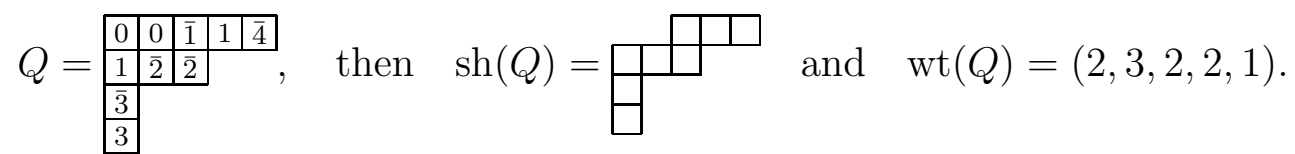

Let

$$
\mathcal{T}_{\left(m_{0}, m_{1}, \ldots, m_{\ell}\right)}^{\lambda}=\left\{\begin{array}{c}
\text { symplectic tableaux of shape } \lambda /\left(m_{0}\right) \\
\text { and weight }\left(m_{0}, m_{1}, \ldots, m_{\ell}\right)
\end{array}\right\} .
$$

A tiling of $\lambda$ by dominoes is a partition of the boxes of $\lambda$ into pairs of adjacent boxes. For example, if

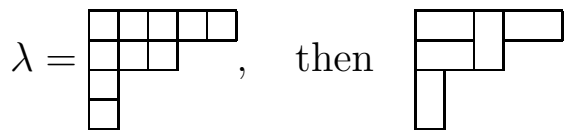

is a tiling of $\lambda$ by dominoes.

Let $\left(m_{0}, m_{1}, \ldots, m_{\ell}\right)$ be a sequence of nonnegative integers such that $m_{0} \leqslant \lambda_{1}$ and $|\lambda|=m_{0}+2\left(m_{1}+\cdots+m_{\ell}\right)$. A domino tableau $Q$ of shape $\lambda /\left(m_{0}\right)=\operatorname{sh}(Q)$ and weight $\left(m_{0}, m_{1}, \ldots, m_{\ell}\right)=\operatorname{wt}(Q)$ is a column strict filling of a tiling of the shape $\lambda /\left(m_{0}\right)$ by dominoes, where if a domino is filled with a number, then that number occupies both boxes covered by that domino. We put 0's in the non-tiled boxes of $\lambda$, and $m_{i}$ is the number of $i$ 's which appear. For example, if

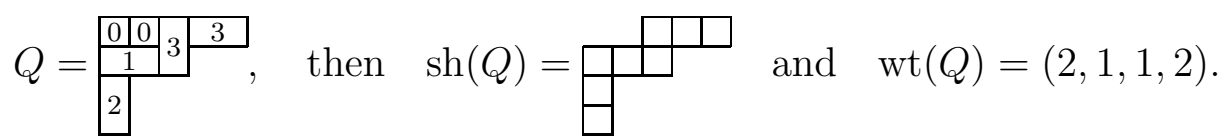

Let

$$
\mathcal{D}_{\left(m_{0}, m_{1}, \ldots, m_{\ell}\right)}^{\lambda}=\left\{\begin{array}{c}
\text { domino tableaux of shape } \lambda /\left(m_{0}\right) \\
\text { and weight }\left(m_{0}, m_{1}, \ldots, m_{\ell}\right)
\end{array}\right\} .
$$

In the following Lemma, (a) is a straightforward use of the usual Pieri rule, and (b) is both similar to (and perhaps a special case of) [14, Theorem 6.3], and also related to a Pieri formula in [12].

Lemma 4.2. Let $\left(m_{0}, m_{1}, \ldots, m_{\ell}\right)$ be an $\ell+1$-tuple of nonnegative integers which sum to $n$. Then

$$
\begin{aligned}
& \text { (a) } s_{\left(m_{0}\right)} \prod_{r=1}^{\ell} \sum_{i=0}^{m_{r}} s_{(i)} s_{\left(m_{r}-i\right)}=\sum_{\lambda \in \mathcal{P}_{n}}\left|\mathcal{T}_{\left(m_{0}, m_{1}, \ldots, m_{\ell}\right)}^{\lambda}\right| s_{\lambda}, \\
& \text { (b) } s_{\left(m_{0}\right)} \prod_{r=1}^{\ell} \sum_{i=0}^{2 m_{r}}(-1)^{i} s_{(i)} s_{\left(2 m_{r}-i\right)}=\sum_{\lambda \in \mathcal{P}_{2 n-m_{0}}}(-1)^{n(\lambda)}\left|\mathcal{D}_{\left(m_{0}, m_{1}, \ldots, m_{\ell}\right)}^{\lambda}\right| s_{\lambda} .
\end{aligned}
$$


Proof. (a) Note that

$$
s_{\left(m_{0}\right)} \prod_{r=1}^{\ell} \sum_{i=0}^{m_{r}} s_{(i)} s_{\left(m_{r}-i\right)}=\sum_{\substack{0 \leqslant i_{r} \leqslant m_{r} \\ 1 \leqslant r \leqslant \ell}} s_{\left(m_{0}\right)} \prod_{r=1}^{\ell} s_{\left(i_{r}\right)} s_{\left(m_{r}-i_{r}\right)}
$$

Now repeated applications of Pieri's rule implies the result.

(b) Note that

$$
s_{\left(m_{0}\right)} \prod_{r=1}^{\ell} \sum_{i=0}^{2 m_{r}}(-1)^{i} s_{(i)} s_{\left(2 m_{r}-i\right)}=\sum_{\substack{0 \leqslant i_{r} \leqslant 2 m_{r} \\ 1 \leqslant r \leqslant \ell}}(-1)^{i_{1}+\cdots+i_{\ell}} s_{\left(m_{0}\right)} \prod_{r=1}^{\ell} s_{\left(i_{r}\right)} s_{\left(2 m_{r}-i_{r}\right)} .
$$

By Pieri's rule,

$$
\sum_{\substack{0 \leqslant i_{r} \leqslant 2 m_{r} \\
1 \leqslant r \leqslant \ell}} s_{\left(m_{0}\right)} \prod_{r=1}^{\ell} s_{\left(i_{r}\right)} s_{\left(2 m_{r}-i_{r}\right)}=\sum_{\lambda \in \mathcal{P}_{2 n-m_{0}}}\left(\begin{array}{c}
\text { Number of column strict fillings of } \lambda \\
\text { using } m_{0} \text { 0's, and for } r=1,2, \ldots, \ell \\
\text { using } i_{r} \bar{r} \text { 's and }\left(2 m_{r}-i_{r}\right) r^{\prime} \text { 's. }
\end{array}\right) s_{\lambda} .
$$

By observing that the sign counts the number of barred entries,

$$
s_{\left(m_{0}\right)} \prod_{r=1}^{\ell} \sum_{i=0}^{2 m_{r}}(-1)^{i} s_{(i)} s_{\left(2 m_{r}-i\right)}=\sum_{\lambda \in \mathcal{P}_{2 n-m_{0}}}\left(\sum_{Q \in \mathcal{T}_{\left(m_{0}, 2 m_{1}, \ldots, 2 m_{\ell}\right)}}(-1)^{\text {Number of barred entries in } Q}\right) s_{\lambda} .
$$

We therefore need to determine the cancellations for a given shape $\lambda$.

Fix $r \in\{1,2, \ldots, \ell\}$ and $\lambda \in \mathcal{P}$ such that $\mathcal{T}_{\left(m_{0}, 2 m_{1}, \ldots, 2 m_{\ell}\right)}^{\lambda} \neq \emptyset$. For a tableau $Q \in$ $\mathcal{T}_{\left(m_{0}, 2 m_{1}, \ldots, 2 m_{\ell}\right)}^{\lambda}$, let

$$
\begin{aligned}
Q_{r} & =\text { skew tableaux consisting of the boxes in } Q \text { containing } \bar{r} \text { or } r, \\
\mathcal{S}_{Q}^{(r)} & =\left\{\text { column strict fillings of } \operatorname{sh}\left(Q_{r}\right) \text { by elements in }\{\bar{r}, r\}\right\} .
\end{aligned}
$$

For example, if

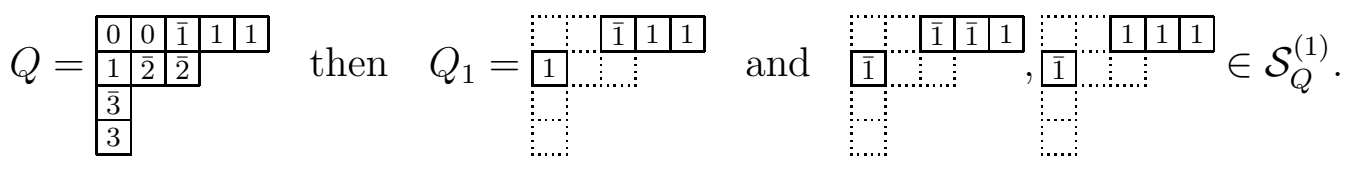

(In fact, $\left.\left|\mathcal{S}_{Q}^{(1)}\right|=8\right)$.

In light of $(4.3)$, (b) is equivalent to

$$
\sum_{Q^{\prime} \in \mathcal{S}_{Q}^{(r)}}(-1)^{\text {Number of } \bar{r} \text { 's in } Q^{\prime}}= \begin{cases}(-1)^{n\left(\operatorname{sh}\left(Q_{r}\right)\right)} & \text { if } \operatorname{sh}\left(Q_{r}\right) \text { has a domino tiling } \\ 0 & \text { otherwise. }\end{cases}
$$


Note that in row $j, Q^{\prime} \in \mathcal{S}_{Q}^{(r)}$ is of the form

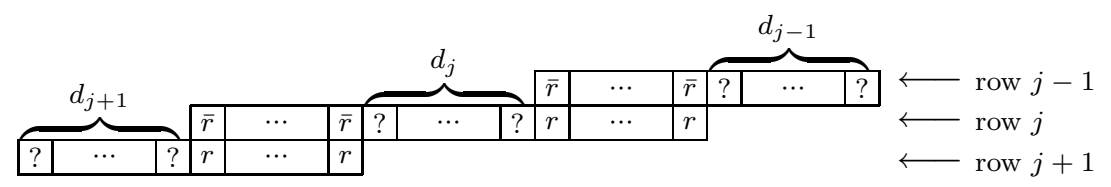

Thus, we have $d_{j}+1$ choices for the values in row $j$. If the total number of choices is even, then exactly half of these choices give a positive sign and half give a negative sign. So we have

$$
\sum_{Q^{\prime} \in \mathcal{S}_{Q}^{(r)}}(-1)^{\text {Number of }} \bar{r} \text { 's in } Q^{\prime}=0,
$$

unless $d_{j}$ is even for all rows $j$. In this case, the signs of all but one of the possible tableaux will cancel each other out, so the only tableau that we have to count has row $j$ of the form

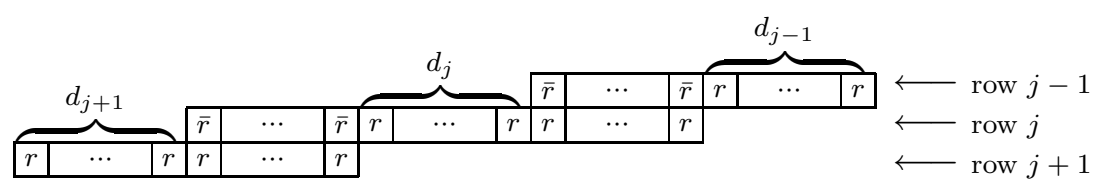

which can clearly be tiled by dominoes of the form $r$ and $r$. For this tableau, we have

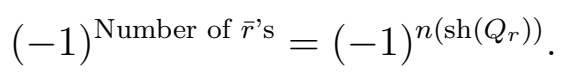

Thus,

$$
s_{\left(m_{0}\right)} \prod_{r=1}^{\ell} \sum_{i=0}^{2 m_{r}}(-1)^{i} s_{(i)} s_{\left(2 m_{r}-i\right)}=\sum_{\lambda \in \mathcal{P}_{2 n-m_{0}}}(-1)^{n(\lambda)}\left|\mathcal{D}_{\left(m_{0}, m_{1}, \ldots, m_{\ell}\right)}^{\lambda}\right| s_{\lambda},
$$

as desired.

Let $\boldsymbol{\lambda} \in \mathcal{P}^{\Theta}$ and $\gamma \in \mathcal{P}^{\Theta}$ be such that $\mathrm{ht}(\boldsymbol{\gamma}) \leqslant 1$ and $\left|\boldsymbol{\gamma}^{(\varphi)}\right| \leqslant \boldsymbol{\lambda}_{1}^{(\varphi)}$ for all $\varphi \in \Theta$. A battery $\Theta$-tableau $\mathbf{Q}$ of shape $\boldsymbol{\lambda} / \boldsymbol{\gamma}$ is a sequence of tableaux indexed by $\Theta$ such that

$$
\mathbf{Q}^{(\varphi)}= \begin{cases}\text { a domino tableau of shape } \boldsymbol{\lambda}^{(\varphi)} / \boldsymbol{\gamma}^{(\varphi)} & \text { if }|\varphi| \text { is odd, } \\ \text { a symplectic tableau of shape } \boldsymbol{\lambda}^{(\varphi)} / \boldsymbol{\gamma}^{(\varphi)} & \text { if }|\varphi| \text { is even }\end{cases}
$$

The weight of $\mathbf{Q}$ is $\operatorname{wt}(\mathbf{Q})=\left(\operatorname{wt}(\mathbf{Q})_{1}\right.$, wt $\left.(\mathbf{Q})_{2}, \ldots\right)$, where

$$
\operatorname{wt}(\mathbf{Q})_{i}=\sum_{\substack{\varphi \in \Theta \\|\varphi| \text { odd }}}|\varphi| \operatorname{wt}\left(\mathbf{Q}^{(\varphi)}\right)_{i}+\sum_{\substack{\varphi \in \Theta \\|\varphi| \text { even }}} \frac{|\varphi|}{2} \operatorname{wt}\left(\mathbf{Q}^{(\varphi)}\right)_{i}
$$

Let

$$
\mathcal{B}_{(k, \nu)}^{\boldsymbol{\lambda}}=\left\{\mathbf{Q} \text { battery tableaux } \mid \operatorname{sh}(\mathbf{Q})=\boldsymbol{\lambda} / \boldsymbol{\gamma}, \boldsymbol{\gamma} \in \mathcal{P}_{k}^{\Theta}, \operatorname{ht}(\boldsymbol{\gamma}) \leqslant 1, \operatorname{wt}(\mathbf{Q})=\nu\right\} .
$$

Example. If

$$
\boldsymbol{\lambda}=\left(\square^{\left(\varphi_{1}\right)}, \square \square^{\left(\varphi_{2}\right)}, \square \square^{\left(\varphi_{3}\right)}\right) \quad \text { where }\left|\varphi_{i}\right|=i
$$


then $\mathcal{B}_{(2,(5,4))}^{\boldsymbol{\lambda}}$ contains

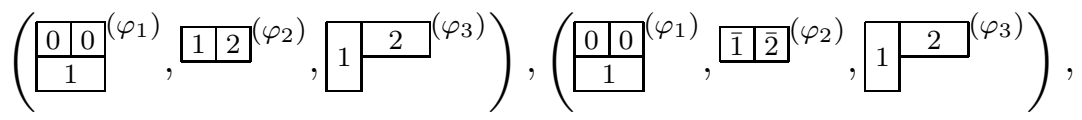

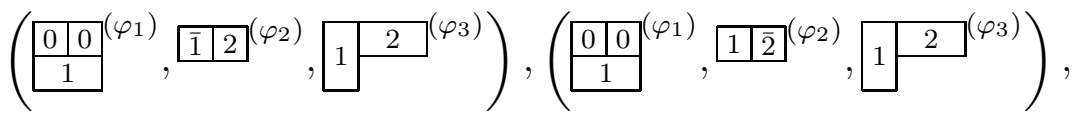

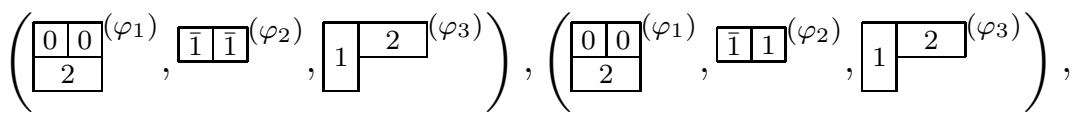

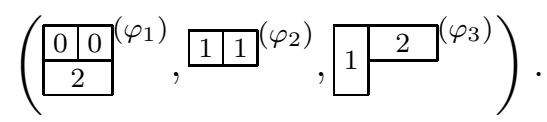

Some intuition. If $\boldsymbol{\lambda} \in \mathcal{P}^{\Theta}$, we can think of the boxes in $\boldsymbol{\lambda}^{(\varphi)}$ as being $|\varphi|$ deep, so in the above example,

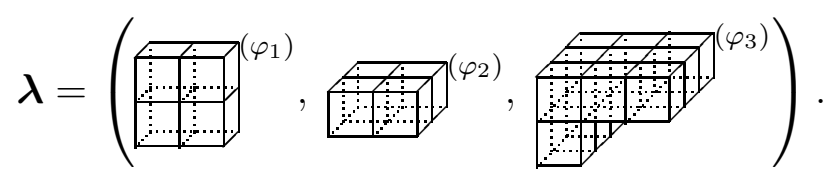

A battery $\Theta$-tableau is a way of stuffing the slots by numbered "batteries" where front and back are distinguished by $i$ and $\bar{i}$, but the sides look generically like $i$, so

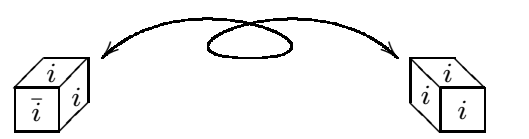

Then a battery $\Theta$-tableau might look like:

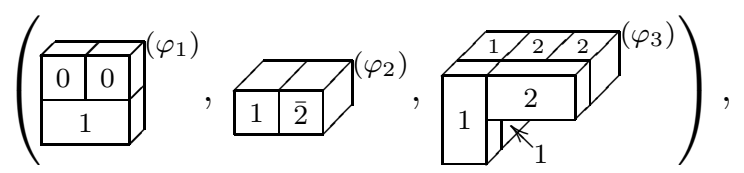

so the weight of the tableau counts the number of batteries of a given type get used, regardless of the cardinality of $\varphi$.

\subsection{Inducing from $G_{n}$ to $U_{2 n}$}

Note that any maximal torus $\tilde{T}_{\nu}$ of $G_{n} \subseteq U_{2 n}$ becomes the maximal torus $T_{2 \nu}$ of $U_{2 n}$, which gives rise to the map

$$
\left.i:\left\{\begin{array}{c}
\text { Pairs }\left(\tilde{T}_{\nu}, \tilde{\theta}_{\nu}\right) \text { with } \tilde{T}_{\nu} \text { a } \\
\operatorname{maximal} \text { torus of } G_{n}, \\
\tilde{\theta}_{\nu} \in \underset{\operatorname{Hom}\left(\tilde{T}_{\nu}, \mathbb{C}^{\times}\right)}{\left(\tilde{T}_{\nu}, \tilde{\theta}_{\nu}\right)}
\end{array}\right\} \mapsto \begin{array}{c}
\text { Pairs }\left(T_{2 \nu}, \theta_{\nu}\right) \text { with } T_{2 \nu} \text { a } \\
\text { maximal torus of } U_{2 n}, \\
\theta_{\nu} \in \operatorname{Hom}\left(T_{2 \nu}, \mathbb{C}^{\times}\right) \\
\left(T_{2 \nu}, \tilde{\theta}_{\nu}\right) .
\end{array}\right\}
$$


To translate the combinatorics between $G_{n}$ and $U_{2 n}$, we define the map

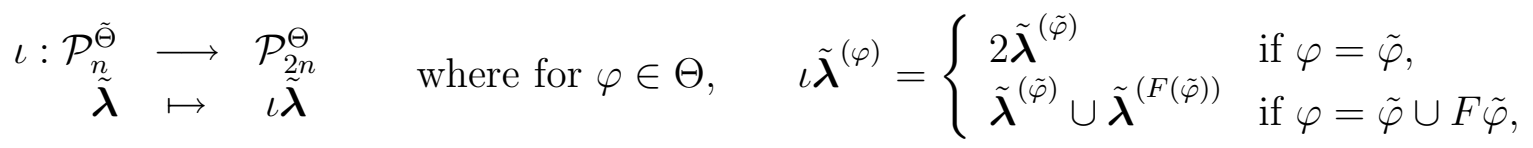

which has the property that $\tau_{\Theta} \circ i=\iota \circ \tau_{\tilde{\Theta}}$ (see (2.12)). The map $\iota$ is neither surjective nor injective. We note that $F \tilde{\varphi}=\tilde{\varphi}$ implies that $|\tilde{\varphi}|$ is odd, and if $F \tilde{\varphi} \neq \tilde{\varphi}$, then $\varphi=\tilde{\varphi} \cup F \tilde{\varphi}$ implies $|\varphi|$ is even (see [5]). Thus, the image of $\iota$ is the set of even $\Theta$-partitions,

$$
\operatorname{Image}(\iota)=\left\{\boldsymbol{\lambda} \in \mathcal{P}_{n}^{\Theta}|| \varphi \mid \boldsymbol{\lambda}^{(\varphi)} \text { is even for } \varphi \in \Theta\right\} \text {. }
$$

Theorem 4.1.

$$
R_{G_{n}}^{U_{2 n}}\left(\tilde{\Gamma}_{(n)}\right)=\sum_{\substack{\boldsymbol{\lambda} \in \mathcal{P}_{2 n}^{\Theta} \\ h t(\boldsymbol{\lambda}) \leqslant 2}}\left|\mathcal{B}_{(0,(n))}^{\boldsymbol{\lambda}}\right| \chi^{\boldsymbol{\lambda}}
$$

Proof. Note that by Theorem 3.1, (2.16), and the characteristic map for $G_{n}$,

$$
\tilde{\Gamma}_{(n)}=\sum_{\substack{\tilde{\boldsymbol{\lambda}} \in \mathcal{P}_{n}^{\tilde{\Theta}} \\ \operatorname{ht}(\tilde{\boldsymbol{\lambda}})=1}} \chi^{\tilde{\boldsymbol{\lambda}}}=\sum_{\substack{\tilde{\boldsymbol{\lambda}} \in \mathcal{P}^{\tilde{\Theta}} \\ \operatorname{ht}(\boldsymbol{\lambda})=1}} \sum_{\substack{\tilde{\boldsymbol{\nu}} \in \mathcal{P}_{s}^{\tilde{\boldsymbol{\lambda}}}\\}} \frac{(-1)^{n-\ell(\tilde{\boldsymbol{\nu}})}}{z_{\tilde{\boldsymbol{\nu}}}} R_{\tilde{\boldsymbol{\nu}}}^{G_{n}}
$$

By transitivity of induction, and the fact that $\tau_{\Theta} \circ i=\iota \circ \tau_{\tilde{\Theta}}$, we have $R_{G_{n}}^{U_{2 n}}\left(R_{\tilde{\boldsymbol{\nu}}}^{G_{n}}\right)=R_{\iota \tilde{\boldsymbol{\nu}}}^{U_{2 n}}$, and so

$$
R_{G_{n}}^{U_{2 n}}\left(\tilde{\Gamma}_{(n)}\right)=\sum_{\substack{\tilde{\boldsymbol{\lambda}} \in \mathcal{P}_{n}^{\tilde{\Theta}} \\ h t(\tilde{\boldsymbol{\lambda}})=1}} \sum_{\tilde{\boldsymbol{\nu}} \in \mathcal{P}_{s}^{\tilde{\boldsymbol{\lambda}}}} \frac{(-1)^{n-\ell(\tilde{\boldsymbol{\nu}})}}{z_{\tilde{\boldsymbol{\nu}}}} R_{\iota \tilde{\boldsymbol{\nu}}}^{U_{2 n}}
$$

We now change the second sum to a sum over $\boldsymbol{\nu}=\iota \tilde{\boldsymbol{\nu}} \in \mathcal{P}_{s}^{\iota \tilde{\boldsymbol{\lambda}}}$, and we obtain

$$
\begin{aligned}
R_{G_{n}}^{U_{2 n}}\left(\tilde{\Gamma}_{(n)}\right)= & \sum_{\substack{\tilde{\boldsymbol{\lambda}} \in \mathcal{P}_{n}^{\tilde{\Theta}} \\
\operatorname{ht}(\tilde{\boldsymbol{\lambda}})=1}} \sum_{\substack{\boldsymbol{\nu} \in \mathcal{P}_{s}^{i \tilde{\lambda}}\\
}}\left(\sum_{\substack{\tilde{\boldsymbol{\nu}} \in \mathcal{P}_{s}^{\tilde{\boldsymbol{\lambda}}} \\
\iota \tilde{\boldsymbol{\nu}}=\boldsymbol{\nu}}} \frac{1}{z_{\tilde{\boldsymbol{\nu}}}}\right)(-1)^{n-\ell(\boldsymbol{\nu})} R_{\boldsymbol{\nu}}^{U_{2 n}} \\
= & \sum_{\substack{\boldsymbol{\nu} \in \mathcal{P}_{2 n}^{\Theta} \\
\boldsymbol{\nu} \text { even }}}\left(\sum_{\substack{\tilde{\boldsymbol{\nu}} \in \mathcal{P}_{\tilde{\Theta}}^{\tilde{\Theta}} \\
\iota \tilde{\boldsymbol{\nu}}=\boldsymbol{\nu}}} \frac{1}{z_{\tilde{\boldsymbol{\nu}}}}\right)(-1)^{n-\ell(\boldsymbol{\nu})} R_{\boldsymbol{\nu}}^{U_{2 n}} .
\end{aligned}
$$

Recall that $F \tilde{\varphi}=\tilde{\varphi}$ implies that $|\tilde{\varphi}|$ is odd, and $F \tilde{\varphi} \neq \tilde{\varphi}$ implies that $\varphi=\tilde{\varphi} \cup F \tilde{\varphi}$ where $|\varphi|$ is even. Apply the characteristic map, factor, and then reindex to obtain

$$
\begin{aligned}
& \operatorname{ch}\left(R_{G_{n}}^{U_{2 n}}\left(\tilde{\Gamma}_{(n)}\right)\right)=(-1)^{n} \sum_{\substack{\boldsymbol{\nu} \in \mathcal{P}_{2 n}^{\Theta} \\
\boldsymbol{\nu} \text { even }}}\left(\sum_{\substack{\tilde{\boldsymbol{\nu}} \in \mathcal{P}^{\Theta} \\
\iota \tilde{\boldsymbol{\nu}}=\boldsymbol{\nu}}} \frac{1}{z_{\tilde{\boldsymbol{\nu}}}}\right) p_{\boldsymbol{\nu}} \\
& =(-1)^{n} \sum_{\substack{\boldsymbol{\nu} \in \mathcal{P}_{2 n}^{\Theta} \\
\boldsymbol{\nu} \text { even }}} \prod_{\substack{\varphi \in \Theta \mid \text { odd } \\
\varphi \in \boldsymbol{\nu}^{(\varphi) / 2}}} \frac{1}{z_{\boldsymbol{\nu}^{(\varphi)}}\left(Y^{(\varphi)}\right)} \prod_{\substack{\varphi \in \Theta \\
|\varphi| \text { even }}}\left(\sum_{\substack{\nu, \mu \in \mathcal{P} \\
\eta \cup \mu=\boldsymbol{\nu}^{(\varphi)}}} \frac{1}{z_{\eta} z_{\mu}}\right) p_{\boldsymbol{\nu}^{(\varphi)}}\left(Y^{(\varphi)}\right)
\end{aligned}
$$




$$
=(-1)^{n} \sum_{\substack{\boldsymbol{\gamma} \in \mathcal{P}_{2 n}^{\Theta} \\ \operatorname{ht}(\boldsymbol{\gamma})=1 \\ \boldsymbol{\gamma} \text { even }}} \prod_{\substack{\varphi \in \Theta \\|\varphi| \text { odd }}}\left(\sum_{\substack{\nu \in \mathcal{P} \\|\nu|=\left|\boldsymbol{\gamma}^{(\varphi)}\right| \\ \nu \text { even }}} \frac{1}{z_{\nu / 2}} p_{\nu}\left(Y^{(\varphi)}\right)\right) \prod_{\substack{\varphi \in \Theta \\|\varphi| \text { even }}}\left(\sum_{\substack{\eta, \mu \in \mathcal{P} \\|\eta|+|\mu|=\left|\boldsymbol{\gamma}^{(\varphi)}\right|}} \frac{1}{z_{\eta} z_{\mu}} p_{\eta \cup \mu}\left(Y^{(\varphi)}\right)\right) .
$$

Note that by (2.1),

$$
\sum_{\substack{\eta, \mu \in \mathcal{P} \\|\eta|+|\mu|=|\gamma|}} \frac{1}{z_{\eta} z_{\mu}} p_{\eta \cup \mu}=\sum_{i=0}^{|\gamma|}\left(\sum_{|\eta|=i} z_{\eta}^{-1} p_{\eta}\right)\left(\sum_{|\mu|=|\gamma|-i} z_{\mu}^{-1} p_{\mu}\right)=\sum_{i=0}^{|\gamma|} s_{(i)} s_{(|\gamma|-i)} .
$$

A computation similar to $[17$, I.2.14] shows that

$$
\sum_{\substack{|\nu|=|\gamma| \\ \nu \text { even }}} \frac{1}{z_{\nu / 2}} p_{\nu}=\sum_{i=0}^{|\gamma|}(-1)^{i} s_{(i)} s_{(|\gamma|-i)}
$$

Thus,

$$
\operatorname{ch}\left(R_{G_{n}}^{U_{2 n}}\left(\tilde{\Gamma}_{(n)}\right)\right)=(-1)^{n} \sum_{\substack{\boldsymbol{\gamma} \in \mathcal{P}_{n}^{\Theta} \\ \text { ht }(\boldsymbol{\gamma})=1 \\ \boldsymbol{\gamma} \text { even }}} \prod_{\varphi \in \Theta} \sum_{i=0}^{\left|\boldsymbol{\gamma}^{(\varphi)}\right|}(-1)^{|\varphi| i} s_{(i)}\left(Y^{(\varphi)}\right) s_{\left(\mid \boldsymbol{\gamma}^{(\varphi) \mid-i)}\right.}\left(Y^{(\varphi)}\right) .
$$

Lemma 4.2 (a) and (b), respectively, imply that

$$
\begin{aligned}
\sum_{i=0}^{k} s_{(i)} s_{(k-i)} & =\sum_{\lambda \in \mathcal{P}_{k}}\left|\mathcal{T}_{(0, k)}^{\lambda}\right| s_{\lambda}, \text { and } \\
\sum_{i=0}^{k}(-1)^{i} s_{(i)} s_{(k-i)} & =\sum_{\lambda \in \mathcal{P}_{k}}(-1)^{n(\lambda)}\left|\mathcal{D}_{(0, k / 2)}^{\lambda}\right| s_{\lambda} .
\end{aligned}
$$

Since $\left|\mathcal{D}_{(0, k / 2)}^{\lambda}\right|=\left|\mathcal{T}_{(0, k)}^{\lambda}\right|=0$ unless $\mathrm{ht}(\lambda) \leqslant 2$,

$$
\begin{aligned}
& \operatorname{ch}\left(R_{G_{n}}^{U_{2 n}}\left(\tilde{\Gamma}_{(n)}\right)\right)
\end{aligned}
$$

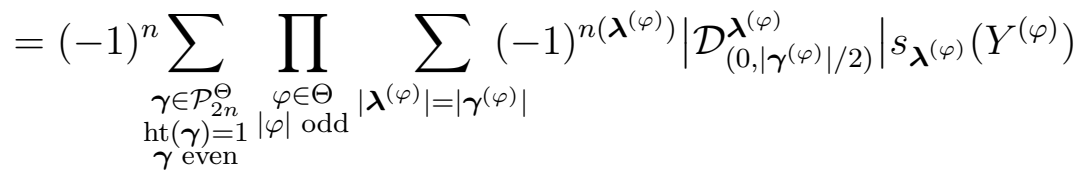

$$
\begin{aligned}
& \cdot \prod_{\substack{\varphi \in \Theta \\
|\varphi| \text { even }}} \sum_{\boldsymbol{\lambda}^{(\varphi)}|=| \boldsymbol{\gamma}^{(\varphi)} \mid}\left|\mathcal{T}_{\left(0,\left|\boldsymbol{\gamma}^{(\varphi)}\right|\right)}^{\boldsymbol{\lambda}^{(\varphi)}}\right| s_{\boldsymbol{\lambda}^{(\varphi)}}\left(Y^{(\varphi)}\right) \\
& =\sum_{\substack{\boldsymbol{\lambda} \in \mathcal{P}_{2 n}^{\Theta} \\
\operatorname{ht}(\boldsymbol{\lambda}) \leqslant 2}}(-1)^{n+n(\boldsymbol{\lambda})}\left|\mathcal{B}_{(0,(n))}^{\boldsymbol{\lambda}}\right| s_{\boldsymbol{\lambda}} .
\end{aligned}
$$

Apply $\mathrm{ch}^{-1}$ to get the result. 
Corollary 4.1. For $n \in \mathbb{Z}_{\geqslant 1}$,

$$
\operatorname{ch}\left(R_{G_{n}}^{U_{2 n}}\left(\tilde{\Gamma}_{(n)}\right)\right)=(-1)^{n} \sum_{\substack{\boldsymbol{\nu} \in \mathcal{P}_{2 n}^{\Theta} \\ \text { ht }(\boldsymbol{\nu})=1 \\ \boldsymbol{\nu} \text { even }}} \prod_{\varphi \in \Theta} \sum_{i=0}^{\left|\boldsymbol{\nu}^{(\varphi)}\right|}(-1)^{i|\varphi|} s_{(i)}\left(Y^{(\varphi)}\right) s_{\left(\left|\boldsymbol{\nu}^{(\varphi)}\right|-i\right)}\left(Y^{(\varphi)}\right) .
$$

Proof. This is (4.5) in the proof of Theorem 4.1.

Using similar techniques, we can prove a result for arbitrary irreducible characters of $G_{n}$. For $\boldsymbol{\lambda} \in \mathcal{P}^{\tilde{\Theta}}$ and $\gamma \in \mathcal{P}_{s}^{i \tilde{\lambda}}$, let

$$
c_{\tilde{\lambda}}^{\gamma}=\prod_{\varphi \in \Theta} c_{\tilde{\boldsymbol{\lambda}}}^{\gamma}(\varphi), \quad \text { where } \quad c_{\tilde{\boldsymbol{\lambda}}}^{\gamma}(\varphi)= \begin{cases}c_{\boldsymbol{\lambda}^{(\tilde{\varphi})}}^{\gamma^{(\varphi)}} & \text { if } \varphi=\tilde{\varphi} \in \tilde{\Theta}, \\ c_{\tilde{\boldsymbol{\lambda}}^{(\tilde{\varphi})} \tilde{\boldsymbol{\lambda}}^{(F \tilde{\varphi})}}^{\gamma^{(\varphi)}} & \text { if } \varphi=\tilde{\varphi} \cup F \tilde{\varphi} \text { and } F \tilde{\varphi} \neq \tilde{\varphi} \in \tilde{\Theta},\end{cases}
$$

where $c_{\nu \mu}^{\lambda}$ is as in (2.4), and $c_{\lambda}^{\gamma}$ is as in (2.5).

Theorem 4.2. Let $\tilde{\boldsymbol{\lambda}} \in \mathcal{P}_{n}^{\tilde{\Theta}}$. Then

$$
R_{G_{n}}^{U_{2 n}}\left(\chi^{\tilde{\boldsymbol{\lambda}}}\right)=\sum_{\gamma \in \mathcal{P}_{s}^{i \tilde{\lambda}}}(-1)^{n(\gamma)} c_{\tilde{\boldsymbol{\lambda}}}^{\gamma} \chi^{\gamma}
$$

Proof. By (2.16) and the characteristic map for $G_{n}$,

$$
\chi^{\tilde{\boldsymbol{\lambda}}}=\sum_{\tilde{\boldsymbol{\nu}} \in \mathcal{P}_{s}^{\tilde{\lambda}}}\left(\prod_{\tilde{\varphi} \in \tilde{\Theta}} \frac{\omega^{\tilde{\boldsymbol{\lambda}}^{(\tilde{\varphi})}\left(\tilde{\boldsymbol{\nu}}^{(\tilde{\varphi})}\right)}}{z_{\tilde{\boldsymbol{\nu}}^{(\tilde{\varphi})}}}\right)(-1)^{n-\ell(\tilde{\boldsymbol{\nu}})} R_{\tilde{\boldsymbol{\nu}}}^{G_{n}} .
$$

By transitivity of induction, and the fact that $\tau_{\Theta} \circ i=\iota \circ \tau_{\tilde{\Theta}}$, we have $R_{G_{n}}^{U_{2 n}}\left(R_{\tilde{\boldsymbol{\nu}}}^{G_{n}}\right)=R_{\iota \tilde{\boldsymbol{\nu}}}^{U_{2 n}}$, and so

$$
R_{G_{n}}^{U_{2 n}}\left(\chi^{\tilde{\boldsymbol{\lambda}}}\right)=\sum_{\tilde{\boldsymbol{\nu}} \in \mathcal{P}_{s}^{\tilde{\boldsymbol{\lambda}}}}\left(\prod_{\tilde{\varphi} \in \tilde{\Theta}} \frac{\omega^{\tilde{\boldsymbol{\lambda}}^{(\tilde{\varphi})}\left(\tilde{\boldsymbol{\nu}}^{(\tilde{\varphi})}\right)}}{z_{\tilde{\boldsymbol{\nu}}(\tilde{\varphi})}}\right)(-1)^{n-\ell(\tilde{\boldsymbol{\nu}})} R_{l \tilde{\boldsymbol{\nu}}}^{U_{2 n}} .
$$

We now change the sum to a sum over $\boldsymbol{\nu}=\iota \tilde{\boldsymbol{\nu}} \in \mathcal{P}_{s}^{i \tilde{\boldsymbol{\lambda}}}$, and using the image of the map $\iota$, we obtain

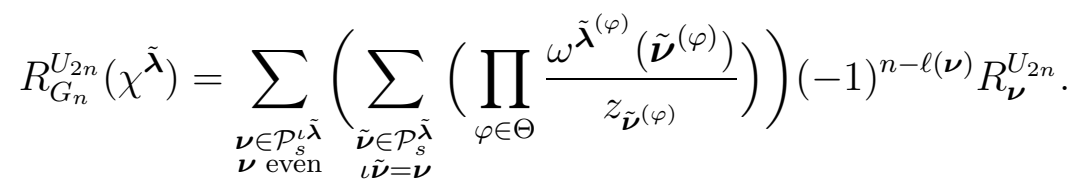

Apply the characteristic map, and rewrite the inner sum and product, to get

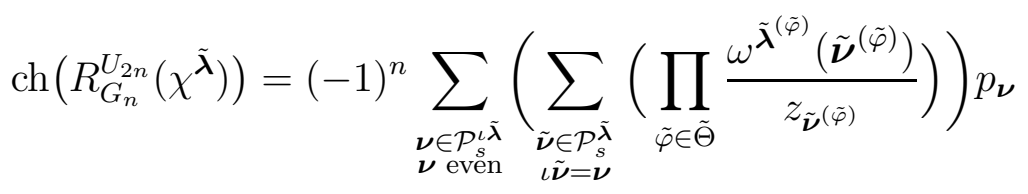




$$
=(-1)^{n} \sum_{\substack{\boldsymbol{\nu} \in \mathcal{P}_{s}^{i \tilde{\lambda}} \\ \boldsymbol{\nu} \text { even }}} \prod_{\substack{\tilde{\varphi} \in \tilde{\Theta} \\ F \tilde{\varphi}=\tilde{\varphi}}} \frac{\omega^{\tilde{\boldsymbol{\lambda}}^{(\tilde{\varphi})}}\left(\boldsymbol{\nu}^{(\tilde{\varphi})} / 2\right)}{z_{\boldsymbol{\nu}(\tilde{\varphi}) / 2}} \prod_{\substack{\tilde{\varphi} \in \tilde{\Theta} \\ F \tilde{\varphi} \neq \tilde{\varphi}}}\left(\sum_{\substack{|\gamma|=\left|\tilde{\boldsymbol{\lambda}}^{(\tilde{\varphi})}\right| \\|\mu|=\left|\tilde{\boldsymbol{\lambda}}^{(F \tilde{\varphi})}\right| \\ \gamma \cup \mu=\boldsymbol{\nu}(\tilde{\varphi} \cup F \tilde{\varphi})}} \frac{\omega^{(\tilde{\boldsymbol{\Lambda}})}(\gamma) \omega^{(F \tilde{\boldsymbol{\varphi}})}(\mu)}{z_{\gamma} z_{\mu}}\right) p_{\boldsymbol{\nu}}
$$

Recall that $F \tilde{\varphi}=\tilde{\varphi}$ implies that $|\tilde{\varphi}|$ is odd, and $F \tilde{\varphi} \neq \tilde{\varphi}$ implies that $\varphi=\tilde{\varphi} \cup F \tilde{\varphi}$ where $|\varphi|$ is even. Thus, for every $\varphi \in \Theta$ such that $\nu^{(\varphi)} \neq \emptyset$, if $|\varphi|$ is odd then $\varphi=\tilde{\varphi}$ for some $\tilde{\varphi} \in \tilde{\Theta}$, and if $|\varphi|$ is even then $\varphi=\tilde{\varphi} \cup F \tilde{\varphi}$ for some $\tilde{\varphi} \in \tilde{\Theta}$. Factor our expression accordingly as

$$
\begin{aligned}
& \operatorname{ch}\left(R_{G_{n}}^{U_{2 n}}\left(\chi^{\tilde{\boldsymbol{\lambda}}}\right)\right)=(-1)^{n} \sum_{\substack{\boldsymbol{\nu} \in \mathcal{P}_{s}^{L \tilde{\boldsymbol{\lambda}}} \\
\boldsymbol{\nu} \text { even }}} \prod_{\varphi=\Theta} \frac{\omega^{(\tilde{\boldsymbol{\lambda}})}\left(\boldsymbol{\nu}^{(\varphi)} / 2\right)}{z_{\boldsymbol{\nu}}^{(\varphi) / 2}} p_{\boldsymbol{\nu}^{(\varphi)}} \prod_{\substack{\varphi \in \Theta \\
\varphi=\tilde{\varphi} \cup F \tilde{\varphi}}}\left(\sum_{\substack{|\gamma|=\left|\tilde{\boldsymbol{\lambda}}^{(\tilde{\varphi})}\right| \mu|=| \tilde{\boldsymbol{\lambda}}^{(F \tilde{\varphi})} \mid \\
\gamma \cup \mu=\boldsymbol{\nu}^{(\varphi)}}} \frac{\omega^{\tilde{\boldsymbol{\lambda}}^{(\tilde{\varphi})}}(\gamma) \omega^{(F \tilde{\boldsymbol{\lambda}})}(\mu)}{z_{\gamma} z_{\mu}}\right) p_{\boldsymbol{\nu}^{(\varphi)}} \\
& =(-1)^{n} \prod_{\substack{\varphi \in \Theta \\
\varphi=\tilde{\varphi}}} \sum_{|\nu|=\left|\tilde{\boldsymbol{\lambda}}^{(\tilde{\varphi})}\right|} \frac{\omega^{\tilde{\boldsymbol{\lambda}}^{(\tilde{\varphi})}}(\nu)}{z_{\nu}} p_{2 \nu}\left(Y^{(\varphi)}\right)
\end{aligned}
$$

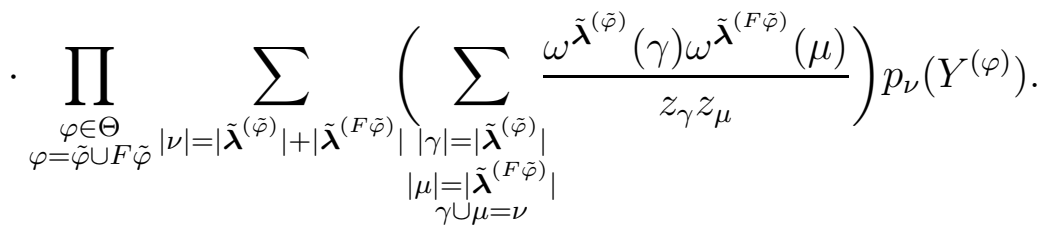

The first product is the case that $|\varphi|$ is odd, and the second product is the case that $|\varphi|$ is even. For the sum in the first product, note that

$$
\begin{aligned}
\left.\sum_{\substack {|\nu|=|\lambda|+|\eta| \\
\begin{subarray}{c}{|\gamma|=|\lambda| \\
|\mu|=|\eta| \\
\gamma \cup \mu=\nu{ | \nu | = | \lambda | + | \eta | \\
\begin{subarray} { c } { | \gamma | = | \lambda | \\
| \mu | = | \eta | \\
\gamma \cup \mu = \nu } }\end{subarray}} \frac{\omega^{\lambda}(\gamma) \omega^{\eta}(\mu)}{z_{\gamma} z_{\mu}}\right) p_{\nu} & =\left(\sum_{|\gamma|=|\lambda|} \frac{\omega^{\lambda}(\gamma)}{z_{\gamma}} p_{\gamma}\right)\left(\sum_{|\mu|=|\eta|} \frac{\omega^{\eta}(\mu)}{z_{\mu}} p_{\mu}\right) \\
& =s_{\lambda} s_{\eta} .
\end{aligned}
$$

For the sum in the product for $|\varphi|$ even, we have

$$
\begin{aligned}
\sum_{|\nu|=|\lambda|} \frac{\omega^{\lambda}(\nu)}{z_{\nu}} p_{2 \nu} & =\sum_{|\nu|=|\lambda|} \frac{\omega^{\lambda}(\nu)}{z_{\nu}} p_{\nu} \circ p_{(2)} \\
& =s_{\lambda} \circ p_{(2)}
\end{aligned}
$$

where $\circ$ is the plethysm product (2.5). Thus, from the definition of the coefficients $c_{\tilde{\boldsymbol{\lambda}}}^{\gamma}$, we have

$$
\operatorname{ch}\left(R_{G_{n}}^{U_{2 n}}\left(\chi^{\tilde{\boldsymbol{\lambda}}}\right)\right)=(-1)^{n} \sum_{\boldsymbol{\gamma} \in \mathcal{P}_{s}^{\iota \tilde{\boldsymbol{\lambda}}}} c_{\tilde{\boldsymbol{\lambda}}}^{\boldsymbol{\gamma}} s_{\boldsymbol{\gamma}}
$$

as desired.

It is perhaps worth noting that since we know Harish-Chandra induction $R_{G_{n}}^{U_{2 n}}\left(\chi^{\tilde{\mathbf{\lambda}}}\right)$ gives a character, then the sign of the coefficient $c_{\tilde{\boldsymbol{\lambda}}}^{\gamma}$ must be $(-1)^{n(\gamma)}$. 


\subsection{Cuspidal characters}

A cuspidal character of $U_{n}$ is an irreducible character $\chi^{\boldsymbol{\lambda}}$ such that, for any Levi subgroup $L$ contained in a proper parabolic subgroup $P \subset U_{n}$, and any character $\chi$ of $L, \chi^{\boldsymbol{\lambda}}$ satisfies $\left\langle R_{L}^{U_{n}}(\chi), \chi^{\boldsymbol{\lambda}}\right\rangle=0$ (see [4, Proposition 6.3]). Note that because of the existence of $P$, the functor $R_{L}^{U_{n}}$ will always be Harish-Chandra induction.

It follows from the description of Levi subgroups in Section 4.2 that every Levi of $U_{n}$ contained in a parabolic is isomorphic to

$$
L \cong G_{k_{1}} \times G_{k_{2}} \times \cdots \times G_{k_{\ell}} \times U_{m},
$$

where $2\left(k_{1}+\cdots+k_{\ell}\right)+m=n$. Thus, the irreducible character of $L$ are indexed by

$$
\left\{\left(\boldsymbol{\lambda}_{1}, \boldsymbol{\lambda}_{2}, \ldots, \boldsymbol{\lambda}_{\ell}, \boldsymbol{\mu}\right) \mid \boldsymbol{\lambda}_{j} \in \mathcal{P}_{k_{j}}^{\tilde{\Theta}}, \boldsymbol{\mu} \in \mathcal{P}_{m}^{\Theta}\right\} .
$$

The characteristic of the corresponding induced character is given by

$$
\operatorname{ch}\left(R_{G_{k_{1}}}^{U_{2 k_{1}}}\left(\chi^{\boldsymbol{\lambda}_{1}}\right)\right) \cdots \operatorname{ch}\left(R_{G_{k_{\ell}}}^{U_{2 k_{\ell}}}\left(\chi^{\boldsymbol{\lambda}_{\ell}}\right)\right) \operatorname{ch}\left(\chi^{\mu}\right) .
$$

Suppose $\boldsymbol{\lambda} \in \mathcal{P}_{k}^{\tilde{\Theta}}$. Define a subgroup $G_{\boldsymbol{\lambda}} \subseteq G_{k}$ by

$$
G_{\boldsymbol{\lambda}}=\prod_{\tilde{\varphi} \in \tilde{\Theta}} G_{\boldsymbol{\lambda}}^{(\tilde{\varphi})}, \quad \text { where } \quad G_{\boldsymbol{\lambda}}^{(\tilde{\varphi})}=G_{|\tilde{\varphi}|}^{\left.\right|^{(\tilde{\varphi})} \mid} .
$$

Note that $G_{\boldsymbol{\lambda}}$ is a Levi of $U_{2 k}$ contained in a parabolic. This parabolic has a distinguished irreducible character $\chi^{[\boldsymbol{\lambda}]}$ given by

$$
\left.\chi^{[\lambda]}\right|_{G_{\lambda}^{(\tilde{\varphi})}}=\underbrace{\chi^{\square^{(\tilde{\varphi})}} \otimes \cdots \otimes \chi^{\square^{(\tilde{\varphi})}}}_{\left|\boldsymbol{\lambda}^{(\tilde{\varphi})}\right| \text { terms }} .
$$

For example, if $k=17$, and

$$
\boldsymbol{\lambda}=\left(\square^{\left(\tilde{\varphi}_{1}\right)}, \square^{\left(\tilde{\varphi}_{2}\right)}, \square^{\left(\tilde{\varphi}_{3}\right)}\right),
$$

where $\left|\tilde{\varphi}_{1}\right|=2,\left|\tilde{\varphi}_{2}\right|=\left|\tilde{\varphi}_{3}\right|=3$. Then $G_{\boldsymbol{\lambda}}=G_{2} \times G_{3} \times G_{3} \times G_{3} \times G_{3} \times G_{3}$, and

$$
[\boldsymbol{\lambda}]=\left(\left(\square^{\left(\tilde{\varphi}_{1}\right)}\right),\left(\square^{\left(\tilde{\varphi}_{2}\right)}\right),\left(\square^{\left(\tilde{\varphi}_{2}\right)}\right),\left(\square^{\left(\tilde{\varphi}_{2}\right)}\right),\left(\square^{\left(\tilde{\varphi}_{3}\right)}\right),\left(\square^{\left(\tilde{\varphi}_{3}\right)}\right)\right) .
$$

The characteristic map for $G_{k}$ sends the Harish-Chandra induced character $R_{G_{\lambda}}^{G_{k}}\left(\chi^{[\lambda]}\right)$ to $\prod_{\tilde{\varphi} \in \tilde{\Theta}}\left(s_{(1)}\left(Y^{(\tilde{\varphi})}\right)\right)^{\left|\lambda^{(\tilde{\varphi})}\right|}$. It follows from Pieri's rules that the inner product

$$
\left\langle R_{G_{\lambda}}^{G_{k}}\left(\chi^{[\boldsymbol{\lambda}]}\right), \chi^{\boldsymbol{\lambda}}\right\rangle=\prod_{\tilde{\varphi} \in \tilde{\Theta}} K_{\left.\boldsymbol{\lambda}^{(\tilde{\varphi}),(1|\lambda(\tilde{\varphi})|}\right)} \neq 0,
$$

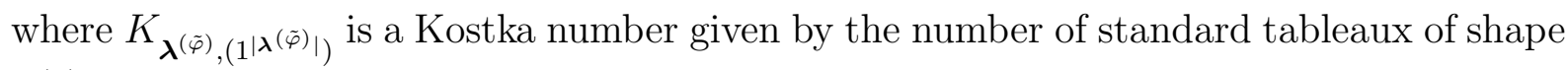
$\boldsymbol{\lambda}^{(\tilde{\varphi})}$ (see [17, Section I.5, Example 2(a)]). Thus, to understand which characters are in the decomposition of characters induced from the split Levis, it suffices to consider the case where $L=G_{k_{1}} \times G_{k_{2}} \times \cdots \times G_{k_{\ell}} \times U_{m}$ and $\chi^{\left(\boldsymbol{\lambda}_{1}, \boldsymbol{\lambda}_{2}, \ldots, \boldsymbol{\lambda}_{\ell}, \boldsymbol{\mu}\right)}$ satisfies

$$
\boldsymbol{\lambda}_{j}=\left(\square^{\left(\tilde{\varphi}_{j}\right)}\right), \quad \text { for some } \tilde{\varphi}_{j} \in \tilde{\Theta} \text { with }\left|\tilde{\varphi}_{j}\right|=k_{j} .
$$


Lemma 4.3. For $\tilde{\varphi} \in \tilde{\Theta}$ with $|\tilde{\varphi}|=k$,

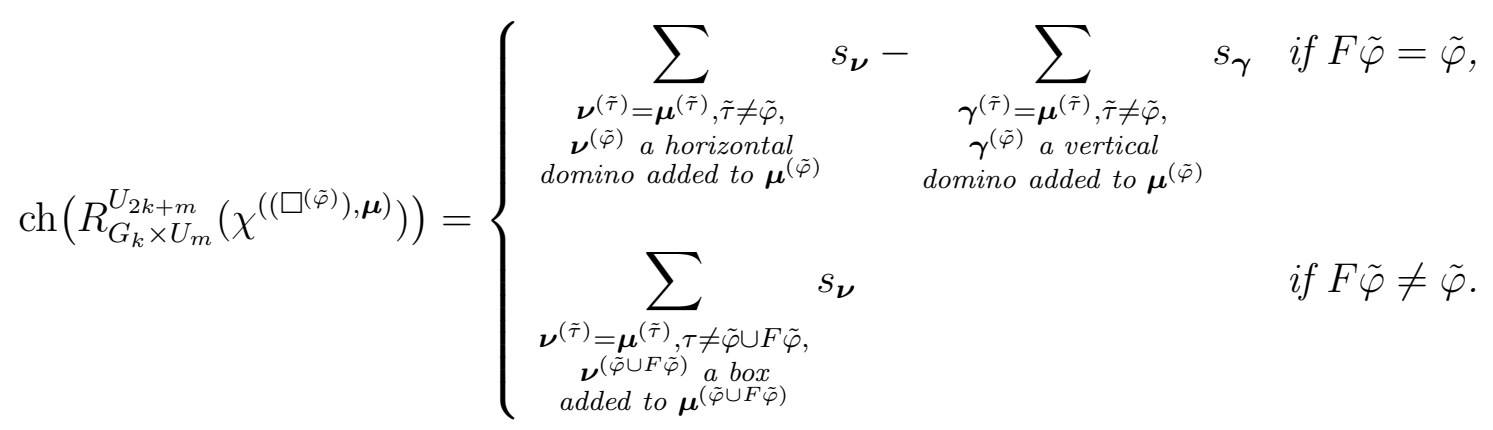

Proof. For $\tilde{\varphi} \in \tilde{\Theta}$ with $|\tilde{\varphi}|=k$, by Theorem 4.2 and the characteristic map for $U_{2 k}$,

$$
\begin{aligned}
\operatorname{ch}\left(R_{G_{k}}^{U_{2 k}}\left(\chi^{\square(\tilde{\varphi})}\right)\right) & = \begin{cases}p_{(2)}\left(Y^{(\tilde{\varphi})}\right) & \text { if } \tilde{\varphi}=F \tilde{\varphi}, \\
s_{(1)}\left(Y^{(\tilde{\varphi} \cup F \tilde{\varphi})}\right) & \text { if } \tilde{\varphi} \neq F \tilde{\varphi},\end{cases} \\
& = \begin{cases}s_{(2)}\left(Y^{(\tilde{\varphi})}\right)-s_{\left(1^{2}\right)}\left(Y^{(\tilde{\varphi})}\right) & \text { if } \tilde{\varphi}=F \tilde{\varphi}, \\
s_{(1)}\left(Y^{(\tilde{\varphi} \cup F \tilde{\varphi})}\right) & \text { if } \tilde{\varphi} \neq F \tilde{\varphi} .\end{cases}
\end{aligned}
$$

Thus,

$$
\operatorname{ch}\left(R_{G_{k} \times U_{m}}^{U_{2 k+m}}\left(\chi^{\left(\left(\square^{(\tilde{\varphi})}\right), \boldsymbol{\mu}\right)}\right)\right)= \begin{cases}\left(s_{(2)}\left(Y^{(\tilde{\varphi})}\right)-s_{\left(1^{2}\right)}\left(Y^{(\tilde{\varphi})}\right)\right) s_{\boldsymbol{\mu}} & \text { if } \tilde{\varphi}=F \tilde{\varphi} \\ s_{(1)}\left(Y^{(\tilde{\varphi} \cup F \tilde{\varphi})}\right) s_{\boldsymbol{\mu}} & \text { if } \tilde{\varphi} \neq F \tilde{\varphi}\end{cases}
$$

The second case follows from usual Pieri rules. The first part follows from the Pieri rules and observing that the common terms in $s_{(2)} s_{\mu}$ and $s_{\left(1^{2}\right)} s_{\mu}$ are exactly those terms that do not come from adding a complete domino (horizontal or vertical).

Let $\mathcal{C}_{2}$ denote the following set of partitions:

$$
\mathcal{C}_{2}=\{(s, s-1, \ldots, 2,1) \in \mathcal{P} \mid s \geqslant 1\}
$$

That is, $\mathcal{C}_{2}$ is the set of "stairstep" partitions, or the 2-cores. These are exactly the partitions from which we may not remove a domino and still have a partition (see Section 5.1). Lusztig proved that the only unipotent characters of any $U_{n}$ which are cuspidal are those which correspond to the partitions $\lambda \in \mathcal{C}_{2}$, and so only occur when $n=s(s+1) / 2=$ $|\lambda|$ for some $s[15$, Propositions 9.2 and 9.4]. We now characterize the set of all cuspidal characters of the finite unitary groups.

Theorem 4.3. The set of all $\boldsymbol{\lambda} \in \mathcal{P}_{n}^{\Theta}$ such that the character $\chi^{\boldsymbol{\lambda}}$ of $U_{n}$ is cuspidal is

$$
\left\{\boldsymbol{\lambda} \in \mathcal{P}_{n}^{\Theta}\left|\boldsymbol{\lambda}=\left(\square^{(\varphi)}\right),\right| \varphi \mid=n\right\} \cup\left\{\boldsymbol{\lambda} \in \mathcal{P}_{n}^{\Theta} \mid \boldsymbol{\lambda}^{(\varphi)} \neq \emptyset \text { implies }|\varphi| \text { is odd and } \boldsymbol{\lambda}^{(\varphi)} \in \mathcal{C}_{2}\right\} .
$$

Proof. Note that by transitivity of Harish-Chandra induction,

$$
\begin{aligned}
R_{G_{k_{1}} \times \cdots \times G_{k_{\ell}} \times U_{m}}^{U_{n}} & \left(\chi^{\left(\left(\square^{\left(\varphi_{1}\right)}\right), \ldots,\left(\square^{\left(\varphi_{\ell}\right)}\right), \boldsymbol{\mu}\right)}\right) \\
& =R_{G_{k_{1}} \times \cdots \times G_{k_{\ell-1}} \times U_{2 k_{\ell}+m}}^{U_{n}}\left(\chi^{\left(\left(\square^{\left(\varphi_{1}\right)}\right), \ldots,\left(\square^{\left(\varphi_{\ell-1}\right)}\right)\right)} \otimes R_{G_{k_{\ell}} \times U_{m}}^{U_{2 k_{\ell}+m}}\left(\chi^{\left(\left(\square^{\left(\varphi_{\ell}\right)}\right), \boldsymbol{\mu}\right)}\right)\right) .
\end{aligned}
$$


We may now iteratively apply Lemma 4.3 to see which $\chi^{\boldsymbol{\lambda}}$ of $U_{n}$ can possibly appear through Harish-Chandra induction. In particular, it follows from Lemma 4.3 that the characters $\chi^{\boldsymbol{\lambda}}$ which cannot appear are exactly those for which $\boldsymbol{\lambda}=\left(\square^{(\varphi)}\right)$ for some $|\varphi|=n$ (so $\lambda$ has only one box); or those for which $\boldsymbol{\lambda}^{(\varphi)}=\emptyset$ when $\varphi=\tilde{\varphi} \cup F \tilde{\varphi}$ for some $\tilde{\varphi} \in \tilde{\Theta}$ and $F \tilde{\varphi} \neq \tilde{\varphi}$ (so $\varphi \notin \tilde{\Theta}$ ), and $\boldsymbol{\lambda}^{(\varphi)}$ has no removable domino (so $\boldsymbol{\lambda}^{(\varphi)} \in \mathcal{C}_{2}$ or $\left.\lambda^{(\varphi)}=\emptyset\right)$ when $\varphi \in \tilde{\Theta}$. Recalling that $\varphi \in \Theta$ satisfies $\varphi \in \tilde{\Theta}$ if and only if $|\varphi|$ is odd, the result follows.

\subsection{Decomposing degenerate Gelfand-Graev characters}

The following theorem is our main theorem of Section 4 .

Theorem 4.4. Let $n \in \mathbb{Z}_{\geqslant 1}$ and let $(k, \nu)$ satisfy $\nu \vdash \frac{n-k}{2} \in \mathbb{Z}_{\geqslant 0}$. Then

$$
\Gamma_{(k, \nu)}=\sum_{\boldsymbol{\lambda} \in \mathcal{P}_{n}^{\Theta}}\left|\mathcal{B}_{(k, \nu)}^{\lambda}\right| \chi^{\boldsymbol{\lambda}}
$$

Proof. Recall that by Proposition 4.1,

$$
\operatorname{ch}\left(\Gamma_{(k, \nu)}\right)=\operatorname{ch}\left(\Gamma_{(k)}\right) \operatorname{ch}\left(R_{G_{\nu_{1}}}^{U_{2 \nu_{1}}}\left(\tilde{\Gamma}_{\left(\nu_{1}\right)}\right)\right) \operatorname{ch}\left(R_{G_{\nu_{2}}}^{U_{2 \nu_{2}}}\left(\tilde{\Gamma}_{\left(\nu_{2}\right)}\right)\right) \cdots \operatorname{ch}\left(R_{G_{\nu_{\ell}}}^{U_{2 \nu_{\ell}}}\left(\tilde{\Gamma}_{\left(\nu_{\ell}\right)}\right)\right)
$$

By Theorem 3.1 and Corollary 4.1,

$$
\begin{aligned}
\operatorname{ch}\left(\Gamma_{(k)}\right) & =(-1)^{\lfloor k / 2\rfloor} \sum_{\substack{\boldsymbol{\gamma} \in \mathcal{P}_{k}^{\Theta} \\
\operatorname{ht}(\boldsymbol{\gamma})=1}} \prod_{\varphi \in \Theta} s_{\boldsymbol{\gamma}^{(\varphi)}}\left(Y^{(\varphi)}\right), \text { and } \\
\operatorname{ch}\left(R_{G_{r}}^{U_{2 r}}\left(\tilde{\Gamma}_{(r)}\right)\right) & =(-1)^{r} \sum_{\substack{\boldsymbol{\gamma} \in \mathcal{P}_{2 r}^{\Theta} \\
\text { ht }(\boldsymbol{\gamma})=1 \\
\boldsymbol{\gamma} \text { even }}} \prod_{\varphi \in \Theta} \sum_{i=0}^{\left|\boldsymbol{\gamma}^{(\varphi)}\right|}(-1)^{i|\varphi|} s_{(i)}\left(Y^{(\varphi)}\right) s_{\left(\left|\boldsymbol{\gamma}^{(\varphi)}\right|-i\right)}\left(Y^{(\varphi)}\right) .
\end{aligned}
$$

Thus,

$$
\operatorname{ch}\left(\Gamma_{(k, \nu)}\right)=(-1)^{\lfloor n / 2\rfloor} \sum_{\substack{\gamma_{0} \in \mathcal{P}_{k}^{\Theta} \\
\operatorname{ht}\left(\boldsymbol{\gamma}_{0}\right)=1}} \sum_{\begin{array}{c}
1 \leqslant r \leqslant \ell(\nu) \\
\boldsymbol{\gamma}_{r} \in \mathcal{P}_{2 \nu r}^{\Theta} \\
\operatorname{ht}\left(\boldsymbol{\gamma}_{r}\right)=1 \\
\boldsymbol{\gamma}_{r} \text { even }
\end{array}} \prod_{\varphi \in \Theta} s_{\gamma_{0}^{(\varphi)}}\left(Y^{(\varphi)}\right) \prod_{r=1}^{\ell(\nu)} \sum_{i=0}^{\left|\boldsymbol{\gamma}_{r}^{(\varphi)}\right|}(-1)^{i|\varphi|} s_{(i)}\left(Y^{(\varphi)}\right) s_{\left(\left|\boldsymbol{\gamma}_{r}^{(\varphi)}\right|-i\right)}\left(Y^{(\varphi)}\right) .
$$

Fix $\varphi \in \Theta$, and let $m_{r}=\left|\boldsymbol{\gamma}_{r}^{(\varphi)}\right|$. If $|\varphi|$ is even, then Lemma 4.2 (a) implies

$$
s_{\left(m_{0}\right)} \prod_{r=1}^{\ell(\nu)} \sum_{i=0}^{m_{r}} s_{(i)} s_{\left(m_{r}-i\right)}=\sum_{|\lambda|=m_{0}+\cdots+m_{\ell(\nu)}}\left|\mathcal{T}_{\left(m_{0}, \ldots, m_{\ell(\nu)}\right)}^{\lambda}\right| s_{\lambda} .
$$


If $|\varphi|$ is odd, then Lemma 4.2 (b) implies

$$
s_{\left(m_{0}\right)} \prod_{r=1}^{\ell(\nu)} \sum_{i=0}^{m_{r}}(-1)^{i} s_{(i)} s_{\left(m_{r}-i\right)}=\sum_{|\lambda|=m_{0}+\cdots+m_{\ell(\nu)}}(-1)^{n(\lambda)}\left|\mathcal{D}_{\left(m_{0}, m_{1} / 2, \ldots, m_{\ell(\nu)} / 2\right)}^{\lambda}\right| s_{\lambda} .
$$

Therefore,

$$
\begin{aligned}
& \operatorname{ch}\left(\Gamma_{(k, \nu)}\right)
\end{aligned}
$$

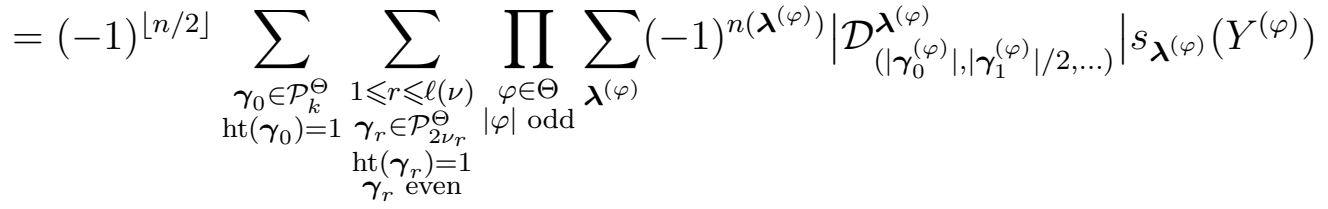

$$
\begin{aligned}
& \cdot \prod_{\substack{\varphi \in \Theta \\
|\varphi| \text { even }}} \sum_{\boldsymbol{\lambda}^{(\varphi)}}\left|\mathcal{T}_{\left(\left|\boldsymbol{\gamma}_{0}^{(\varphi)}\right|,\left|\boldsymbol{\gamma}_{1}^{(\varphi)}\right| \ldots\right)}^{\boldsymbol{\lambda}^{(\varphi)}}\right| s_{\boldsymbol{\lambda}^{(\varphi)}}\left(Y^{(\varphi)}\right) \\
& =(-1)^{\lfloor n / 2\rfloor} \sum_{\boldsymbol{\lambda} \in \mathcal{P}_{n}^{\Theta}}\left(\sum_{\substack{\boldsymbol{\gamma}_{0} \in \mathcal{P}_{k}^{\Theta} \\
\operatorname{ht}\left(\boldsymbol{\gamma}_{0}\right)=1}} \sum_{\substack{1 \leqslant r \leqslant \ell(\nu) \\
\boldsymbol{\gamma}_{r} \in \mathcal{P}_{2 \nu r}^{\Theta} \\
\text { ht }\left(\boldsymbol{\gamma}_{r}\right)=1 \\
\boldsymbol{\gamma}_{r} \text { even }}} \prod_{\substack{\varphi \in \Theta \\
\text { edd }}}(-1)^{n\left(\boldsymbol{\lambda}^{(\varphi)}\right)}\left|\mathcal{D}_{\left(\left|\boldsymbol{\gamma}_{0}^{(\varphi)}\right|,\left|\boldsymbol{\gamma}_{1}^{(\varphi)}\right| / 2, \ldots\right)}^{\boldsymbol{\lambda}^{(\varphi)}}\right| \prod_{\substack{\varphi \in \Theta \\
|\varphi| \text { even }}}\left|\mathcal{T}_{\left(\left|\boldsymbol{\gamma}_{0}^{(\varphi)}\right|,\left|\boldsymbol{\gamma}_{1}^{(\varphi)}\right| \ldots\right)}^{\boldsymbol{\lambda}^{(\varphi)}}\right|\right) s_{\boldsymbol{\lambda}} \\
& =\sum_{\boldsymbol{\lambda} \in \mathcal{P}_{n}^{\Theta}}(-1)^{\lfloor n / 2\rfloor+n(\boldsymbol{\lambda})}\left|\mathcal{B}_{(k, \nu)}^{\boldsymbol{\lambda}}\right| s_{\boldsymbol{\lambda}} .
\end{aligned}
$$

The result follows by applying $\mathrm{ch}^{-1}$.

\section{Some multiplicity consequences}

In this section we explore some of the multiplicity implications of Theorem 4.4.

\subsection{A bijection between domino tableaux and pairs of column strict tableaux}

The 2-core of a partition $\lambda \in \mathcal{P}$, which we denote $\operatorname{core}_{2}(\lambda)$, is the partition of minimal size such that the skew partition $\lambda / \operatorname{core}_{2}(\lambda)$ may be tiled by dominoes. It is not difficult to see that the 2 -core of any partition is always of the form $(m, m-1, \ldots, 2,1)$ for some nonnegative integer $m$ (where (0) is the empty partition).

The 2-quotient quot $_{2}(\lambda)$ of a partition $\lambda$ is a pair of partitions $\left(\operatorname{quot}_{2}(\lambda)^{(0)}, \operatorname{quot}_{2}(\lambda)^{(1)}\right)$ (defined in [17, I.1, Example 8]). We define

$$
\operatorname{quot}_{2}(\lambda)_{i}=\operatorname{quot}_{2}(\lambda)_{i}^{(0)}+\operatorname{quot}_{2}(\lambda)_{i}^{(1)}
$$

Also define the content of a box $\square$ in the $i$ th row and $j$ th column of a partition $\lambda$ to be $j-i$. 
Let $\lambda \in \mathcal{P}_{n}$ with $\operatorname{core}_{2}(\lambda) \in\{(0),(1)\}$. Consider the bijection

$$
\begin{gathered}
\mathcal{D}_{\left(\left|\operatorname{core}_{2}(\lambda)\right|, m_{1}, \ldots, m_{\ell}\right)}^{\lambda} \\
Q
\end{gathered} \longleftrightarrow\left\{\begin{array}{c}
\text { Pairs of column strict } \\
\text { tableaux of shape } \text { quot }_{2}(\lambda) \\
\text { and weight }\left(m_{1}, m_{2}, \ldots, m_{\ell}\right)
\end{array}\right\}
$$

given by the following algorithm, which originally appeared in [21], and is in a more general form in [13].

(1) Each domino in $Q$ covers two boxes of $\lambda / \operatorname{core}_{2}(\lambda)$. Move the entries in $Q$ to the box that has content 0 modulo 2.

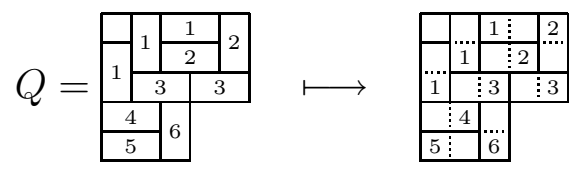

(2) Let $\mathcal{S}^{(0)}$ denote the set of all dominoes that have the entry in the lower or leftmost box, and $\mathcal{S}^{(1)}$ be the set of dominoes that have the entry in the upper or rightmost box.

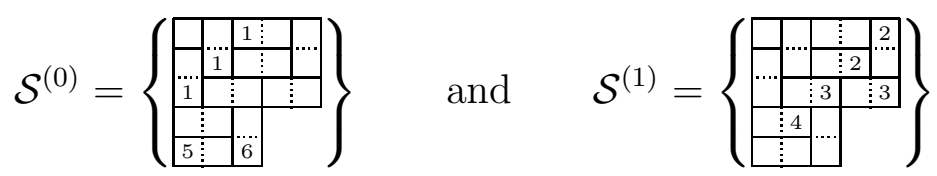

(3) For even $-\ell(\lambda)<i<\lambda_{1}$ and $j \in\{0,1\}$, let

$$
\begin{gathered}
D_{i}^{(j)}=\begin{array}{l}
\text { The increasing sequence of entries whose } \\
\text { content is } i \text { and whose domino is in } \mathcal{S}^{(j)},
\end{array} \\
\left(D_{-4}^{(0)}, D_{-2}^{(0)}, D_{0}^{(0)}, D_{2}^{(0)}, D_{4}^{(0)}\right)=((5),(1,6),(1),(1),()) \\
\left(D_{-4}^{(1)}, D_{-2}^{(1)}, D_{0}^{(1)}, D_{2}^{(1)}, D_{4}^{(1)}\right)=((),(4),(3),(2,3),(2)) .
\end{gathered}
$$

(4) Let $Q^{(j)}$ be the unique tableau that has increasing diagonal sequences given by the $D_{i}^{(j)}$ for all even $-\ell(\lambda)<i<\lambda_{1}$.

$$
Q^{(0)}=\begin{array}{l|l|l}
1 & 1 & 1 \\
\hline 5 & 6
\end{array} \quad \text { and } \quad Q^{(1)}=\begin{array}{lll}
2 & 2 \\
\hline 3 & 3 \\
\hline 4
\end{array}
$$

\section{Remarks.}

1. If the shape of the domino tableau $Q$ is $\lambda / \operatorname{core}_{2}(\lambda)$, then the shape of $\left(Q^{(0)}, Q^{(1)}\right)$ is $\operatorname{quot}_{2}(\lambda)$. 
2. We may apply this algorithm to a domino tableau of shape $\lambda /(m)$ with $m \equiv$ $\left|\operatorname{core}_{2}(\lambda)\right| \bmod 2$, by requiring that the tableau of shape $\lambda / \operatorname{core}_{2}(\lambda)$ has $\lfloor m / 2\rfloor$ horizontal dominoes filled with zeroes. For example,

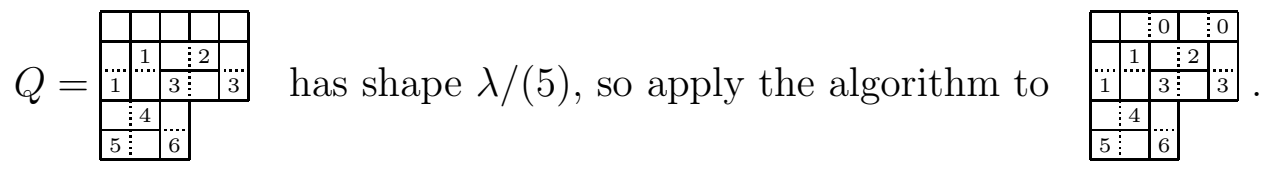

Note that all of the zero dominoes are in the same set $\mathcal{S}^{\left(\left|\operatorname{core}_{2}(\lambda)\right|\right)}$, so changing $m$ corresponds to adding or subtracting the number of zeroes in the first row of $Q^{\left(\left|\operatorname{core}_{2}(\lambda)\right|\right)}$.

We will use the lexicographic total ordering on partitions given by

$\lambda \leqslant \mu$ if there exists $k \in \mathbb{Z}_{\geqslant 1}$ such that $\lambda_{k}<\mu_{k}$ and $\lambda_{i}=\mu_{i}$ for $1 \leqslant i<k$.

Lemma 5.1. Let $\lambda \in \mathcal{P}_{n}$ be such that $\operatorname{core}_{2}(\lambda) \in\{(0),(1)\}$, and let $0 \leqslant m \leqslant \lambda_{1}$ be such that $m \equiv\left|\operatorname{core}_{2}(\lambda)\right| \bmod 2$. Then there exists a lexicographically maximal weight $\mu=\left(\mu_{1}, \mu_{2}, \ldots, \mu_{\ell}\right)$ such that there exists exactly one domino tableau of shape $\lambda /(m)$ and weight $\left(m, \mu_{1}, \ldots, \mu_{\ell}\right)$.

Proof. First suppose $\left(\lambda^{(0)} / \gamma^{(0)}, \lambda^{(1)} / \gamma^{(1)}\right)$ is a pair of skew partitions. Let $\mu_{1}$ be the maximal number of 1's we can put in a tableau of shape $\left(\lambda^{(0)} / \gamma^{(0)}, \lambda^{(1)} / \gamma^{(1)}\right), \mu_{2}$ be the maximal number of 2's we can thereafter fill into $\left(\lambda^{(0)} / \gamma^{(0)}, \lambda^{(1)} / \gamma^{(1)}\right)$, and $\mu_{j}$ be the maximal number of $j$ 's we can fill given that we have filled in a maximum number at each step up to $j$. Then there is exactly one tableau $\left(Q^{(0)}, Q^{(1)}\right)$ of shape $\left(\lambda^{(0)} / \gamma^{(0)}, \lambda^{(1)} / \gamma^{(1)}\right)$ and weight $\mu$, and this weight is lexicographically maximal. The result now follows from pulling back $\left(Q^{(0)}, Q^{(1)}\right)$ through the bijection (5.1) to get a domino tableau of the same weight, along with the second remark preceding this Lemma.

Remark. If $m=\left|\operatorname{core}_{2}(\lambda)\right|$, then $\mu$ is given by $\mu_{0}=\left|\operatorname{core}_{2}(\lambda)\right|$ and $\mu_{i}=\operatorname{quot}_{2}(\lambda)_{i}$ for $i \geqslant 1$.

\subsection{Multiplicity results}

As a first consequence of Theorem 4.4, Corollary 5.1, below, characterizes which irreducible characters of $\mathrm{U}\left(n, \mathbb{F}_{q^{2}}\right)$ appear with nonzero multiplicity in some degenerate Gelfand-Graev character. We note that the following could also be obtained by the description of such characters given by Kotlar in [11, Corollary 2.6] based on Harish-Chandra series.

Corollary 5.1. The set of all $\boldsymbol{\lambda} \in \mathcal{P}_{n}^{\Theta}$ such that the character $\chi^{\boldsymbol{\lambda}}$ of $\mathrm{U}\left(n, \mathbb{F}_{q^{2}}\right)$ satisfies $\left\langle\chi^{\lambda}, \Gamma_{(k, \nu)}\right\rangle \neq 0$ for some degenerate Gelfand-Graev character $\Gamma_{(k, \nu)}$ is

$$
\left\{\boldsymbol{\lambda} \in \mathcal{P}_{n}^{\Theta} \mid \operatorname{core}_{2}\left(\boldsymbol{\lambda}^{(\varphi)}\right) \in\{(0),(1)\} \text { whenever }|\varphi| \text { is odd }\right\} .
$$


Proof. By Theorem 4.4, the irreducible character $\chi^{\boldsymbol{\lambda}}$ appears with nonzero multiplicity in some degenerate Gelfand-Graev character if and only if there is a battery tableau of shape $\boldsymbol{\lambda} / \boldsymbol{\gamma}$ for some $\boldsymbol{\gamma} \in \mathcal{P}^{\Theta}$ with $\operatorname{ht}(\boldsymbol{\gamma}) \leqslant 1$.

If for some odd $\varphi \in \Theta$, we have $\operatorname{core}_{2}\left(\boldsymbol{\lambda}^{(\varphi)}\right) \notin\{(0),(1)\}$, then the 2-core of $\boldsymbol{\lambda}^{(\varphi)}$ has at least two parts. But then there is no choice of $\gamma^{(\varphi)}$ that allows us to tile $\lambda^{(\varphi)} / \gamma^{(\varphi)}$ by dominoes. On the other hand, if $\operatorname{core}_{2}\left(\boldsymbol{\lambda}^{(\varphi)}\right)=(0)$, we can choose $\boldsymbol{\gamma}^{(\varphi)}=(0)$, and if core $_{2}\left(\boldsymbol{\lambda}^{(\varphi)}\right)=(1)$, we can let $\boldsymbol{\gamma}^{(\varphi)}=(1)$, and $\boldsymbol{\lambda}^{(\varphi)} / \boldsymbol{\gamma}^{(\varphi)}$ can be tiled by dominoes.

We now specify multiplicities of certain characters $\chi^{\boldsymbol{\lambda}}$ in degenerate Gelfand-Graev characters.

Theorem 5.1. Let $\boldsymbol{\lambda} \in \mathcal{P}_{n}^{\Theta}$ be such that $\operatorname{core}_{2}\left(\boldsymbol{\lambda}^{(\varphi)}\right) \in\{(0),(1)\}$ whenever $|\varphi|$ is odd. Then there exists $\nu \vdash \frac{n-k}{2}$ such that

$$
\left\langle\Gamma_{(k, \nu)}, \chi^{\boldsymbol{\lambda}}\right\rangle=\prod_{\substack{\varphi \in \Theta \\|\varphi| \text { even }}} \prod_{i \text { odd }}\left(\boldsymbol{\lambda}_{i}^{(\varphi)}-\boldsymbol{\lambda}_{i+1}^{(\varphi)}+1\right) .
$$

Proof. Let $k=\sum_{|\varphi| \text { odd }}|\varphi|\left|\operatorname{core}_{2}\left(\boldsymbol{\lambda}^{(\varphi)}\right)\right|$ and define $\gamma$ by

$$
\gamma^{(\varphi)}= \begin{cases}\operatorname{core}_{2}\left(\boldsymbol{\lambda}^{(\varphi)}\right) & \text { if }|\varphi| \text { is odd } \\ \emptyset & \text { otherwise }\end{cases}
$$

Since $|\gamma|=k$, by Theorem 4.4 and Corollary 5.1, it suffices to find $\nu \vdash \frac{n-k}{2}$ such that there exist

$$
\prod_{\substack{\varphi \in \Theta \\|\varphi| \text { even }}} \prod_{i \text { odd }}\left(\boldsymbol{\lambda}_{i}^{(\varphi)}-\boldsymbol{\lambda}_{i+1}^{(\varphi)}+1\right)
$$

battery tableaux with shape $\boldsymbol{\lambda} / \boldsymbol{\gamma}$ and weight $(k, \nu)$.

We construct the battery tableau $\mathbf{Q}$ as follows. For odd $\varphi \in \Theta$, let $\mathbf{Q}^{(\varphi)}$ be the unique domino tableau of shape $\boldsymbol{\lambda}^{(\varphi)} /\left(\operatorname{core}_{2}\left(\boldsymbol{\lambda}^{(\varphi)}\right)\right)$ and weight

$$
\left(\left|\operatorname{core}_{2}\left(\boldsymbol{\lambda}^{(\varphi)}\right)\right|, \operatorname{quot}_{2}\left(\boldsymbol{\lambda}^{(\varphi)}\right)_{1}, \operatorname{quot}_{2}\left(\boldsymbol{\lambda}^{(\varphi)}\right)_{2}, \ldots\right),
$$

obtained from Lemma 5.1 (see, in particular, the remark after the lemma).

For even $\varphi \in \Theta$ and for each $i \geqslant 1$, we fill the $(2 i-1)$ st row of $\boldsymbol{\lambda}^{(\varphi)}$ with $\bar{i}$ 's, and the $2 i$ th row with $i$ 's. The resulting symplectic tableau $\mathbf{Q}^{(\varphi)}$ has weight $\left(\boldsymbol{\lambda}_{1}^{(\varphi)}+\boldsymbol{\lambda}_{2}^{(\varphi)}, \boldsymbol{\lambda}_{3}^{(\varphi)}+\boldsymbol{\lambda}_{4}^{(\varphi)}, \ldots\right)$. Note that we may change up to $\boldsymbol{\lambda}_{2 i-1}^{(\varphi)}-\boldsymbol{\lambda}_{2 i}^{(\varphi)}$ of the $\bar{i}$ 's to $i$ 's in row $2 i-1$ while leaving the weight unchanged. We therefore have exactly

$$
\prod_{i \text { odd }}\left(\boldsymbol{\lambda}_{i}^{(\varphi)}-\boldsymbol{\lambda}_{i-1}^{(\varphi)}+1\right)
$$

symplectic tableaux of shape $\boldsymbol{\lambda}^{(\varphi)}$ and weight $\left(\boldsymbol{\lambda}_{1}^{(\varphi)}+\boldsymbol{\lambda}_{2}^{(\varphi)}, \boldsymbol{\lambda}_{3}^{(\varphi)}+\boldsymbol{\lambda}_{4}^{(\varphi)}, \ldots\right)$. 
We combine these to create a battery tableau of shape $\boldsymbol{\lambda} / \boldsymbol{\gamma}$ and weight $\nu$, where

$$
\nu_{i}=\sum_{\substack{\varphi \in \Theta \\|\varphi| \text { odd }}}|\varphi| \operatorname{quot}_{2}\left(\boldsymbol{\lambda}^{(\varphi)}\right)_{i}+\sum_{\substack{\varphi \in \Theta \\|\varphi| \text { even }}} \frac{|\varphi|}{2}\left(\boldsymbol{\lambda}_{2 i}^{(\varphi)}+\boldsymbol{\lambda}_{2 i-1}^{(\varphi)}\right)
$$

Note that from this construction, $\nu$ is the maximal weight under the lexicographical ordering (5.2) of a battery tableau of shape $\boldsymbol{\lambda} / \boldsymbol{\gamma}$, while each $\boldsymbol{\gamma}^{(\varphi)}$ is chosen minimally. It follows that the weight $\nu$ will change if we change the weight of any $\mathbf{Q}^{(\varphi)}$.

For example, if

$$
\boldsymbol{\lambda}=\left(\square^{\left(\varphi_{1}\right)}, \square^{\left(\varphi_{2}\right)}, \square^{\left(\varphi_{3}\right)}\right) \quad \text { where }\left|\varphi_{i}\right|=i
$$

then $k=1, \nu=(2+4+3,0+1+3)=(9,4)$, and every battery tableau of shape $\boldsymbol{\lambda}$ and weight $(k, \nu)$ must be of the form

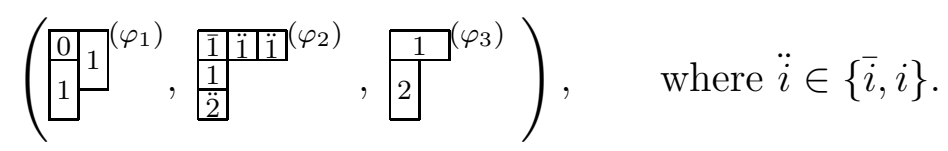

There are $3 \cdot 2=6$ such tableaux.

Theorem 5.1 and its proof give the following multiplicity one result.

Corollary 5.2. Let $\boldsymbol{\lambda} \in \mathcal{P}_{n}^{\Theta}$. Suppose $\operatorname{core}_{2}\left(\boldsymbol{\lambda}^{(\varphi)}\right) \in\{(0),(1)\}$ whenever $|\varphi|$ is odd, and for all $i>0$, the multiplicities $m_{i}\left(\boldsymbol{\lambda}^{(\varphi)}\right)$ are even for all even $\varphi \in \Theta$. Then $\left\langle\Gamma_{(k, \nu)}, \chi^{\boldsymbol{\lambda}}\right\rangle=1$, where

$$
k=\sum_{\substack{\varphi \in \Theta \\|\varphi| \text { odd }}}|\varphi|\left|\operatorname{core}_{2}\left(\boldsymbol{\lambda}^{(\varphi)}\right)\right| \quad \text { and } \quad \nu_{i}=\sum_{\substack{\varphi \in \Theta \\|\varphi| \text { odd }}}|\varphi| \operatorname{quot}_{2}\left(\boldsymbol{\lambda}^{(\varphi)}\right)_{i}+\sum_{\substack{\varphi \in \Theta \\|\varphi| \text { even }}} \frac{|\varphi|}{2}\left(\boldsymbol{\lambda}_{2 i}^{(\varphi)}+\boldsymbol{\lambda}_{2 i-1}^{(\varphi)}\right) .
$$

The next theorem generalizes Corollary 5.2.

Theorem 5.2. Suppose $\boldsymbol{\lambda} \in \mathcal{P}_{n}^{\Theta}$ satisfies the following:

(a) Whenever $|\varphi|$ is odd, $\operatorname{core}_{2}\left(\boldsymbol{\lambda}^{(\varphi)}\right) \in\{(0),(1)\}$,

(b) Whenever $|\varphi|$ is even, the partition $\boldsymbol{\lambda}^{(\varphi)}$ has at most one nonzero part with odd multiplicity,

(c) There exists an $r>0$ such that for every $\varphi \in \Theta$ with $|\varphi|$ even, either $\ell\left(\boldsymbol{\lambda}^{(\varphi)}\right)<r$ and $\boldsymbol{\lambda}^{(\varphi)}$ has no nonzero part of odd multiplicity, or $\boldsymbol{\lambda}_{r}^{(\varphi)}$ has odd multiplicity and $\boldsymbol{\lambda}_{r}^{(\varphi)}<\boldsymbol{\lambda}_{r-1}^{(\varphi)}$.

Then the irreducible character $\chi^{\boldsymbol{\lambda}}$ of $U_{n}$ appears with multiplicity one in some degenerate Gelfand-Graev character of $U_{n}$. 
Proof. Suppose $\boldsymbol{\lambda} \in \mathcal{P}_{n}^{\Theta}$ satisfies (a), (b), and (c). Let $\boldsymbol{\gamma} \in \mathcal{P}^{\Theta}$ be given by

$$
\gamma^{(\varphi)}= \begin{cases}(i) & \text { if }|\varphi| \text { is even and } m_{i}\left(\boldsymbol{\lambda}^{(\varphi)}\right) \text { is odd } \\ \left(\left|\operatorname{core}_{2}(\lambda)\right|+2 \operatorname{quot}_{2}(\lambda)_{\lceil r / 2\rceil}^{\left(\left|\operatorname{core}_{2}(\lambda)\right|\right)}\right) & \text { if }|\varphi| \text { is odd and } \lambda=\boldsymbol{\lambda}^{(\varphi)}\end{cases}
$$

For example, the $\Theta$-partition

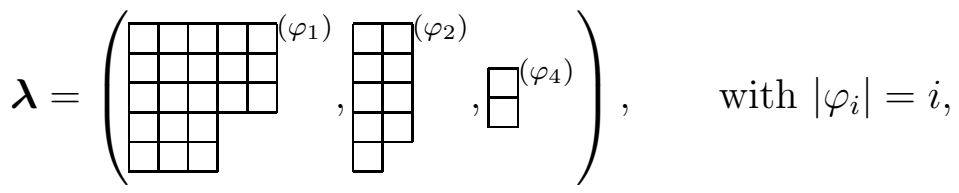

satisfies (a), (b), and (c) with $r=5$. Since

$$
\operatorname{quot}_{2}\left(\boldsymbol{\lambda}^{\left(\varphi_{1}\right)}\right)=\left(\square^{(0)}, \square^{(1)}\right) \quad \text { and } \quad \operatorname{core}_{2}\left(\boldsymbol{\lambda}^{\left(\varphi_{1}\right)}\right)=(1)
$$

(as in the example for (5.1)), we have that

$$
\left|\operatorname{core}_{2}\left(\boldsymbol{\lambda}^{\left(\varphi_{1}\right)}\right)\right|+2 \operatorname{quot}_{2}\left(\boldsymbol{\lambda}^{\left(\varphi_{1}\right)}\right)_{\lceil r / 2\rceil}^{(1)}=3 \quad \text { and } \quad \gamma=\left(\square \square \square^{\left(\varphi_{1}\right)}, \square^{\left(\varphi_{2}\right)}\right) .
$$

Consider the following battery tableau $\mathrm{Q}$ of shape $\boldsymbol{\lambda} / \boldsymbol{\gamma}$.

1. For $\varphi \in \Theta$ such that $|\varphi|$ is even, fill $\boldsymbol{\lambda}^{(\varphi)} / \boldsymbol{\gamma}^{(\varphi)}$ with $\boldsymbol{\lambda}_{2 j-1}^{(\varphi)} j$ 's and $\boldsymbol{\lambda}_{2 j}^{(\varphi)} \bar{j}$ 's for $2 j \leqslant \ell\left(\boldsymbol{\lambda}^{(\varphi)}\right)$ such that $2 j<r$, and $\boldsymbol{\lambda}_{2 j}^{(\varphi)} j$ 's and $\boldsymbol{\lambda}_{2 j+1}^{(\varphi)} \bar{j}$ 's for $2 j+1 \leqslant \ell\left(\boldsymbol{\lambda}^{(\varphi)}\right)$ such that $2 j>r$. Then all of the nonzero entries come in pairs $\frac{j}{j}$ and the resulting weight is lexicographically maximal.

2. For $\varphi \in \Theta$ such that $|\varphi|$ is odd, use Lemma 5.1 to fill $\boldsymbol{\lambda}^{(\varphi)} / \boldsymbol{\gamma}^{(\varphi)}$ in a lexicographically maximal way.

In our running example, we have

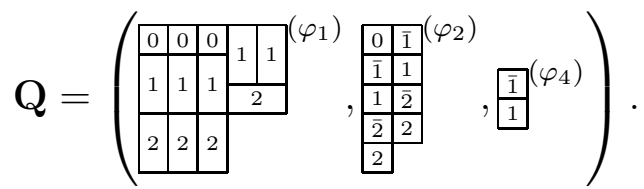

Note that by Lemma 5.1, Q is the only battery tableau of shape $\boldsymbol{\lambda} / \boldsymbol{\gamma}$ and weight wt $(\mathbf{Q})$. Thus, it suffices to show that there is no $\boldsymbol{\nu} \subseteq \boldsymbol{\lambda}$ with $|\boldsymbol{\nu}|=|\gamma|$ and ht $(\boldsymbol{\nu}) \leqslant 1$ such that there exists a battery tableau $\mathbf{P}$ of shape $\boldsymbol{\lambda} / \boldsymbol{\nu}$ and weight wt $(\mathbf{Q})$.

Since $|\boldsymbol{\nu}|=|\boldsymbol{\gamma}|$, we may think of moving from $\mathbf{Q}$ to $\mathbf{P}$ by shifting zero entries between $\varphi \in \Theta$ in $\mathbf{Q}$. If $|\varphi|$ is even, it is clear from the construction of $\mathbf{Q}^{(\varphi)}$ that if we add a zero, an entry $<r / 2$ is lost, while if we remove a zero, an entry $>r / 2$ is gained. Now consider when $|\varphi|$ is odd, with $Q=\mathbf{Q}^{(\varphi)}$ and $\lambda=\boldsymbol{\lambda}^{(\varphi)}$. Apply the bijection (5.1) to the domino tableau $Q$, and notice that from Remark 2 preceding Lemma 5.1, our choice of $\gamma^{(\varphi)}$ forces $Q^{\left(\left|\operatorname{core}_{2}(\lambda)\right|\right)}$ to have exactly quot $_{2}(\lambda)_{\lceil r / 2\rceil}^{\left(\operatorname{core}_{2}(\lambda)\right)}$ 0's. Now, adding a pair of zero 
entries to or removing a pair of zero entries from $Q$ is the same as adding a zero to or removing a zero from $Q^{\left(\left|\operatorname{core}_{2}(\lambda)\right|\right)}$. It is clear that adding a zero to $Q^{\left(\left|\operatorname{core}_{2}(\lambda)\right|\right)}$ results in losing an entry $<r / 2$, which removes a domino with entry $<r / 2$ in $Q$, and removing a zero from $Q^{\left(\left|\operatorname{core}_{2}(\lambda)\right|\right)}$ results in gaining an entry $>r / 2$, which adds a domino with entry $>r / 2$ in $Q$. Thus, no matter how we change $\boldsymbol{\gamma}$ to $\boldsymbol{\nu}$, we are forced to change the weight of the full battery tableau to a lexicographically smaller weight. So, there is no such $\boldsymbol{\nu}$ which leaves the weight unchanged, and uniqueness follows.

Remarks. Corollary 5.2 follows from Theorem 5.2, since (a) and (b) are easily satisfied, and

$$
r=\max \left\{\ell\left(\boldsymbol{\lambda}^{(\varphi)}\right)|\varphi \in \Theta,| \varphi \mid \text { even }\right\}+1 .
$$

Another consequence of Theorem 5.2 is a result by Ohmori [18].

Corollary 5.3 (Ohmori). Let $\boldsymbol{\lambda} \in \mathcal{P}_{n}^{\Theta}$, and define the partition $\mu$ to have parts

$$
\mu_{j}=\sum_{\varphi \in \Theta}|\varphi| \boldsymbol{\lambda}_{j}^{(\varphi)}
$$

Suppose that $\mu=\left(1^{m_{1}} 2^{m_{2}} \ldots\right)$ is such that $m_{i}$ is even for all $i$ except for the one value $i=k$, or that $m_{i}$ is always even, in which case we let $k=0$. Define the partition $\nu$ to be $\nu=\left(1^{m_{1} / 2} 2^{m_{2} / 2} \cdots k^{\left(m_{k}-1\right) / 2} \cdots\right)$. Then the irreducible character $\chi^{\boldsymbol{\lambda}}$ appears with multiplicity one in the degenerate Gelfand-Graev character $\Gamma_{(k, \nu)}$.

Proof. Note that if $\boldsymbol{\lambda} \in \mathcal{P}_{n}^{\Theta}$ satisfies the hypotheses of the corollary, then for any $\varphi \in \Theta$ the partition $\boldsymbol{\lambda}^{(\varphi)}$ has at most one nonzero part size with odd multiplicity, otherwise $\mu$ would have more parts with odd multiplicity. Thus, $\boldsymbol{\lambda}$ satisfies condition (b) of Theorem 5.2. Moreover, the fact that $\mu$ has at most one part with odd multiplicity implies that there must be an $r>0$ such that for every $\varphi \in \Theta$, either $\ell\left(\boldsymbol{\lambda}^{(\varphi)}\right)<r$ or $\boldsymbol{\lambda}_{r}^{(\varphi)}$ has odd multiplicity in $\boldsymbol{\lambda}^{(\varphi)}$ and $\boldsymbol{\lambda}_{r}^{(\varphi)}<\boldsymbol{\lambda}_{r-1}^{(\varphi)}$. In particular, this holds when $|\varphi|$ is even, and so $\boldsymbol{\lambda}$ satisfies condition (c) of Theorem 5.2. If $\varphi \in \Theta$ is odd, and $\boldsymbol{\lambda}^{(\varphi)}$ has a part size $i$ with odd multiplicity, where $i=0$ if $\ell\left(\boldsymbol{\lambda}^{(\varphi)}\right)<r$, then

$$
\operatorname{core}_{2}\left(\boldsymbol{\lambda}^{(\varphi)}\right)= \begin{cases}(1) & \text { if } i \text { is odd } \\ (0) & \text { if } i \text { is even }\end{cases}
$$

Thus, $\boldsymbol{\lambda}$ satisfies (a) of Theorem 5.2.

Now define $\boldsymbol{\gamma}$ by $\boldsymbol{\gamma}^{(\varphi)}=i$ if $m_{i}\left(\boldsymbol{\lambda}^{(\varphi)}\right)$ is odd, where $i=0$ if $\ell\left(\boldsymbol{\lambda}^{(\varphi)}\right)<r$. Then $|\boldsymbol{\gamma}|=k$. When $|\varphi|$ is even, fill $\boldsymbol{\lambda}^{(\varphi)} / \gamma^{(\varphi)}$ just as in the proof of Theorem 5.2. When $|\varphi|$ is odd, fill $\boldsymbol{\lambda}^{(\varphi)} / \boldsymbol{\gamma}^{(\varphi)}$ with all vertical dominoes such that there are $\boldsymbol{\lambda}_{2 j-1}^{(\varphi)} j$ 's for $2 j-1 \leqslant \ell\left(\boldsymbol{\lambda}^{(\varphi)}\right)$ such that $2 j-1<r$, and $\boldsymbol{\lambda}_{2 j}^{(\varphi)} j$ 's for $2 j \leqslant \ell\left(\boldsymbol{\lambda}^{(\varphi)}\right)$ such that $2 j>r$. This gives a battery tableau of shape $\boldsymbol{\lambda} / \boldsymbol{\gamma}$, where $|\boldsymbol{\gamma}|=k$, and weight $\nu$ as defined above.

Fix a $\varphi$ such that $|\varphi|$ is odd, let $\lambda=\boldsymbol{\lambda}^{(\varphi)}$, and let $\mathbf{Q}^{(\varphi)}=Q$ be the domino tableau just defined, and apply the bijection (5.1) to $Q$. Since $Q$ has been filled with all vertical dominoes, the resulting weight is lexicographically maximal, and so by Lemma 5.1, the 
tableaux $Q^{(0)}$ and $Q^{(1)}$ obtained from the bijection (5.1) also have lexicographically maximal weights. Let $j=\left|\operatorname{core}_{2}(\lambda)\right|$, and consider $Q^{(j)}$. From our choice of $\gamma^{(\varphi)}$, the tableau $Q^{(j)}$ has exactly $\left\lfloor\lambda_{r} / 2\right\rfloor 0$ 's. By the bijection (5.1), we also have row $\lceil r / 2\rceil$ of $Q^{(j)}$, which is quot $_{2}\left(\lambda^{(\varphi)}\right)_{[r / 2\rceil}^{(j)}$, is exactly $\left\lfloor\lambda_{r} / 2\right\rfloor$. This means the domino tableau $\mathbf{Q}^{(\varphi)}$ is exactly what is constructed in the proof of Theorem 5.2. Therefore, $\mathrm{Q}$ is exactly the battery tableau obtained in the proof of Theorem 5.2, and so we have $\left\langle\chi^{\boldsymbol{\lambda}}, \Gamma_{(k, \nu)}\right\rangle=1$.

Note that by Corollary 5.1, condition (a) of Theorem 5.2 is a necessary condition. The following proposition shows that condition (b) is also necessary.

Proposition 5.1. Let $\boldsymbol{\lambda} \in \mathcal{P}^{\Theta}$. If there exists a $\varphi \in \Theta$ such that $|\varphi|$ is even and $\boldsymbol{\lambda}^{(\varphi)}$ has at least two distinct part sizes with odd multiplicity, then

$$
\left\langle\chi^{\lambda}, \Gamma_{(k, \nu)}\right\rangle \neq 1
$$

for all degenerate Gelfand-Graev characters $\Gamma_{(k, \nu)}$.

Proof. Suppose $\boldsymbol{\lambda} \in \mathcal{P}^{\Theta}$ and $|\varphi|$ is even, such that $\lambda=\boldsymbol{\lambda}^{(\varphi)}$ has part sizes $x<y$ with odd multiplicity. Let $Q$ be a symplectic tableau of shape $\lambda /(m)$ for some $m \leqslant \lambda_{1}$, and suppose $\operatorname{wt}(Q)=\mu$. If there exists an $\bar{i}$ such that there is no $i$ directly south of $\bar{i}$ in $Q$, then, taking the $\bar{i}$ furthest to the right in this row, there is a second symplectic tableau $P$ of shape $\lambda /(m)$ and weight $\mu$ obtained by changing this $\bar{i}$ to an $i$ in $Q$. Similarly, if there is an $i$ with no $\bar{i}$ directly north of it, then there is a second tableau $P$ with the same weight and shape as $Q$. Thus, the only way $Q$ is the only tableau of shape $\lambda /(m)$ and weight $\mu$, is if $\lambda /(m)$ can be tiled by vertical dominoes.

If $m<y$, then the $y$ th column of $\lambda /(m)$ has an odd number of boxes, and therefore cannot be tiled by vertical dominoes. If $m>y$, then the $m$ th column of $\lambda /(m)$ has an odd number of boxes. If $m=y$, then the $x$ th column of $\lambda /(m)$ has an odd number of boxes. In all cases, $\lambda /(m)$ cannot be tiled by dominoes, and the result follows.

\section{Remarks.}

1. While conditions (a) and (b) of Theorem 5.2 are necessary, condition (c) is not. For example, the only battery tableau of weight $(2,(8))$ for the $\Theta$-partition

$$
\boldsymbol{\lambda}=\left(\square^{(\alpha)}, \square^{(\beta)}\right) \quad \text { with }|\alpha|=4,|\beta|=2, \text { is } \quad \mathbf{Q}=\left(\frac{\square}{1}^{(\alpha)}, \square^{(\beta)}\right)
$$

2. At the same time, conditions (a) and (b) of Theorem 5.2 are not alone sufficient. For example,

$$
\boldsymbol{\lambda}=\left(\square^{(\alpha)}, \square^{(\beta)}\right), \quad \text { with }|\alpha|=|\beta|=2,
$$

satisfies (a) and (b). The possible weights and two of their battery tableaux are

$$
\left.(0,(6)): \quad\left(\square^{(1)}\right)^{(\alpha)}, \square^{(\beta)}\right), \quad\left(\frac{1}{1}^{(\alpha)}, \square^{(\beta)}\right) \quad(2 \text { total }),
$$




$$
\begin{aligned}
& (0,(4,2)): \quad\left(\frac{1}{1}^{(\alpha)}, \square^{(\beta)}\right), \quad\left(\frac{1}{1}^{(\alpha)}, 2^{(\beta)}\right) \quad \text { (10 total) } \\
& \left(0,\left(2^{3}\right)\right): \quad\left(\frac{1}{2}^{(\alpha)}, \square^{(\beta)}\right), \quad\left(\frac{1}{2}^{(\alpha)}, 3^{(\beta)}\right) \quad \text { (24 total), } \\
& (2,(4)): \quad\left(\square^{\frac{0}{1}}{ }^{(\alpha)}, \square^{(\beta)}\right), \quad\left(\square^{\frac{0}{1}}{ }^{(\alpha)}, \square^{(\beta)}\right) \quad(5 \text { total }), \\
& \left(2,\left(2^{2}\right)\right): \quad\left(\square_{\overline{1}}^{(\alpha)}, \square^{(\beta)}\right), \quad\left(\square^{0}{ }^{(\alpha)}, 2^{(\beta)}\right) \quad \text { (12 total), } \\
& (4,(2)): \quad\left(\square^{(\alpha)}, \square^{(\beta)}\right), \quad\left(\frac{0}{1}^{(\alpha)}, \square^{(\beta)}\right) \quad \text { (2 total). }
\end{aligned}
$$

\section{References}

[1] R. Carter, Finite groups of Lie type: conjugacy classes and complex characters. John Wiley and Sons, 1985.

[2] Y. M. Chen, A. M. Garsia, and J. Remmel, Algorithms for plethysm, In: Combinatorics and algebra, Boulder, Colo., 1983, In: Contemp. Math., vol. 13, Amer. Math. Soc., Providence, RI, 1984, pp. 109-153.

[3] P. Deligne and G. Lusztig, Representations of reductive groups over finite fields, Ann. of Math. (2) 103 (1976), no. 1, 103-161.

[4] F. Digne and J. Michel, Foncteurs de Lusztig et caractères des groupes linéaires et unitaires sur un corps fini, J. Algebra 107 (1987), no. 1, 217-255.

[5] V. Ennola, On the conjugacy classes of the finite unitary groups, Ann. Acad. Sci. Fenn. Ser. A I No. 313 (1962), 13 pages.

[6] V. Ennola, On the characters of the finite unitary groups, Ann. Acad. Sci. Fenn. Ser. A I No. 323 (1963), 35 pages.

[7] I. M. Gelfand and M. I. Graev, Construction of irreducible representations of simple algebraic groups over a finite field, Dokl. Akad. Nauk SSSR 147 (1962), 529-532.

[8] R. Hotta and T. A. Springer, A specialization theorem for certain Weyl group representations and an application to the Green polynomials of unitary groups, Invent. Math. 41 (1977), no. 2, 113-127.

[9] R. B. Howlett and C. Zworestine, On Klyachko's model for the representations of finite general linear groups, In Representations and quantizations (Shanghai, 1998), pages 229-245, China High. Educ. Press, Beijing, 2000.

[10] N. Kawanaka, Generalized Gel'fand-Graev representations and Ennola duality, In Algebraic groups and related topics (Kyoto/Nagoya, 1983), 175-206, Adv. Stud. Pure Math., 6, North-Holland, Amsterdam, 1985.

[11] D. Kotlar, On the irreducible components of degenerate Gelfand-Graev characters, J. Algebra 173 (1995), no. 2, 248-260.

[12] T. Lam, Ribbon tableaux and the Heisenberg algebra, Math. Z. 250 (2005), 685-710.

[13] A. Lascoux, B. Leclerc, and J.-Y. Thibon, Ribbon tableaux, Hall-Littlewood functions, and unipotent varieties, Sém. Lothar. Combin. 34 (1995), 23 pages. 
[14] B. Leclerc and J.-Y. Thibon, Littlewood-Richardson coefficients and KazhdanLusztig polynomials. Combinatorial methods in representation theory (Kyoto, 1998), 155-220, Adv. Stud. Pure Math., 28, Kinokuniya, Tokyo, 2000.

[15] G. Lusztig, Irreducible representations of finite classical groups, Invent. Math. 43 (1977), no. 2, 125-175.

[16] G. Lusztig and B. Srinivasan, The characters of the finite unitary groups, J. Algebra 49 (1977), no. 1, 167-171.

[17] I. G. Macdonald, Symmetric functions and Hall polynomials. Second edition. With Contributions by A. Zelevinsky. Oxford Mathematical Monographs. Oxford Science Publications. The Clarendon Press, Oxford University Press, New York, 1995.

[18] Z. Ohmori, On a Zelevinsky theorem and the Schur indices of the finite unitary groups, J. Math. Sci. Univ. Tokyo 2 (1997), no. 2, 417-433.

[19] J. G. Rainbolt, The generalized Gelfand-Graev representations of U(3, q), J. Algebra 202 (1998), no. 1, 44-71.

[20] B. Srinivasan, The characters of the finite symplectic group $S p(4, q)$, Trans. Amer. Math. Soc. 131 (1968), 488-525.

[21] D. W. Stanton and D. E. White, A Schensted algorithm for rim hook tableaux, J. Comb. Theory Ser. A 40 (1985), no. 2, 211-247.

[22] R. Steinberg, Lectures on Chevalley groups, mimeographed notes, Yale University, 1968.

[23] N. Thiem and C. R. Vinroot, On the characteristic map of finite unitary groups, Adv. Math. 210 (2007), no. 2, 707-732.

[24] N. Thiem and C. R. Vinroot, Values of character sums for finite unitary groups, $J$. Algebra 320 (2008), no. 3, 1150-1173.

[25] T. Yokonuma, Sur le commutant d'une représentation d'un groupe de Chevalley fini, J. Fac. Sci. Univ. Tokyo Sec. I 15 (1968), 115-129.

[26] T. Yokonuma, Complément au mémoire "Sur le commutant d'une représentation d'un groupe de Chevalley fini", J. Fac. Sci. Univ. Tokyo Sect. I 16 (1969), 147-148.

[27] A. V. Zelevinsky, Representations of finite classical groups. A Hopf algebra approach, Lecture Notes in Mathematics 869, Springer-Verlag, Berlin-New York, 1981. 